\title{
Recovery and nonrecovery of the untrained state in an exchange-coupled system
}

\author{
Jaru Jutimoosik, ${ }^{1,2}$ Rattikorn Yimnirun, ${ }^{2}$ Annette Setzer, ${ }^{3}$ Pablo Esquinazi, ${ }^{3}$ Jochen Stahn, ${ }^{4}$ and Amitesh Paul ${ }^{1, *}$ \\ ${ }^{1}$ Technische Universität München, Physik Department, Lehrstuhl für Neutronenstreuung, James-Franck-Strasse 1, \\ D-85748 Garching b. München, Germany \\ ${ }^{2}$ School of Physics, Institute of Science and NANOTEC-SUT Center of Excellence on Advanced Functional Nanomaterials, \\ Suranaree University of Technology, Nakhon Ratchasima 30000, Thailand \\ ${ }^{3}$ Division of Superconductivity and Magnetism, University of Leipzig, D-04103 Leipzig, Germany \\ ${ }^{4}$ Laboratory for Neutron Scattering Paul Scherrer Institut, CH-5232 Villigen, Switzerland \\ (Received 23 February 2015; revised manuscript received 6 May 2015; published 25 June 2015)
}

\begin{abstract}
We report depth sensitive investigations of the magnetic interaction between exchange-coupled stacked $\mathrm{CoO}$ and ferromagnetic Co bilayers (separated by thick Au layers) as we explore the degree of recovery of the untrained state after the first two field cycles. Such a recovery is expected by field cycling a reorientation field $\left(H_{\mathrm{RE}}\right)$ along a direction $\left(\Omega_{\mathrm{RE}}\right)$ away from the initial field cooling direction. Measurements as a function of $\Omega_{\mathrm{RE}}$ and the strength of $H_{\mathrm{RE}}$ (along each direction) map the influence of $\Omega_{\mathrm{RE}}$ on the reversal mechanism in the layers and thereby the degree of recovery. Our results are consistent with the earlier observations in similar systems that was realized with $\Omega_{\mathrm{RE}}=90^{\circ}$. We ascribe these partial and/or significant recoveries to the unchanged sense of rotation after initial field cooling of the ferromagnetic magnetization upon each field cycling. Furthermore, in our system, we find that this recovery can be regulated by choosing various other $H_{\mathrm{RE}}$ and $\Omega_{\mathrm{RE}}$ values without changing the rotational sense. The best recipe for recovery is identified for $\Omega_{\mathrm{RE}}=45^{\circ}$, that can be achieved partially with $H_{\mathrm{RE}}=3.0 \mathrm{kOe}$ and remain significant even with $H_{\mathrm{RE}}=10.0 \mathrm{kOe}$. In this study we not only understand the fundamental mechanism in the recovery of training, but also instigate its technological prospects by lifting the directional restrictions of the reorientation field.
\end{abstract}

DOI: 10.1103/PhysRevB.91.224428

PACS number(s): 61.05.fj, 75.70.Cn, 75.60.Jk

\section{INTRODUCTION}

Interfacial exchange coupling between a ferromagnet (FM) and an antiferromagnet (AF) can "lock" the magnetization into the FM in a well-defined direction. This effect, which in a phenomenological picture takes the form of a unidirectional magnetic anisotropy, is known as exchange bias [1]. A cooling field $H_{\mathrm{FC}}$ (cooling below the ordering temperature of $\mathrm{AF}$ ) essentially determines the state of the FM which in-turn determines the strength of the bias field $H_{\mathrm{EB}}$ [2-4].

We focus on a polycrystalline Co (ferromagnet)/CoO (antiferromagnet) exchange bias system. It is ideal for the investigation due to its large biasing field [5], very distinct asymmetry of magnetization reversal [2], large enough training effects [6], and most interestingly the AF moment configuration can be frozen-in in a variety of ways during the process of field cooling [7] without affecting the overall structure as the AF ordering temperature is far below room temperature (leading to negligible interdiffusion at the interfaces). The difference between subsequent (partial) magnetization reversal loops is called the "training effect." The training effect can be linked to a deviation of the average interfacial magnetization vector of the AF $\mathrm{CoO}$ grains with respect to the initial field cooling direction. Even though the microscopic origin of the training effect is still under debate, it is generally agreed to be due to some initial nonequilibrium arrangement leading to such a metastable state of the AF spins [5,8-11]. Therefore, it is perceived that the metastable state can somehow be rearranged to reinduce the original state.

\footnotetext{
*Corresponding author: amitesh.paul@frm2.tum.de
}

Brems et al. [11] attempted to restore the untrained state, i.e., the untrained state with its pronounced asymmetry was largely reinduced by a moderate magnetic field, applied perpendicular $\left(\perp^{r}\right)$ to the initial cooling field in a $\mathrm{Co} / \mathrm{CoO}$ bilayer system. In fact a very similar phenomenon was reported earlier by the same group on stripes of $\mathrm{CoO} / \mathrm{Co}$ as well [12]. The only difference being the strength of the cooling field that may determine the degree of asymmetry in reversal in the latter version. Very recently, Brems et al. [13] have reported of tracking the average rotation sense of the magnetization vector of a $20 \mathrm{~nm}$ Co layer upon magnetization reversal in a similar $\mathrm{CoO} / \mathrm{Co}$ bilayer system. Their inference on the rotational sense relied upon the anisotropic magnetoresistence (AMR) signal measured along $45^{\circ}$ with respect to the cooling field and also the $\perp^{r}$ field directions.

It may be noted that the AMR measurements rely on the current distribution within the sample and are difficult to infer on any reinduction of the untrained state within a multilayered system, particularly when domains are involved. AMR values are weak when a sample consists of different domains of equal proportions that can rotate clockwise or counterclockwise. Moreover, the direction of the reorienting magnetic fields (with different magnitudes) in the works of Brems et al. were always restricted along or opposite to the direction $\perp^{r}$ to the initial cooling field.

Using depth sensitive polarized neutron reflectivity (PNR), Paul et al. $[14,15]$ reported earlier that such a partial restoration of the untrained state was indeed possible even within a $\mathrm{Co} / \mathrm{CoO} / \mathrm{Au}$ multilayer. Since the bilayers of the multilayer was separated by a thick nonmagnetic Au layer at each AF-FM interface, it was expected to behave as a repeated stack of independent $\mathrm{Co} / \mathrm{CoO}$ bilayers. Interestingly, the PNR data showed that the $\perp^{r}$ field cycling has inflicted an additional 
modification at the $\mathrm{Co} / \mathrm{CoO}$ interface magnetization. One may note that PNR is sensitive to each and every interface along the depth of the whole multilayer stack and to the direction of the parallel magnetic moment (though not to the directional sense of the perpendicular magnetic moment). However, such a conclusion on interface magnetism in the work of Paul et al. suffered from two shortcomings. First, the PNR data were limited to a relatively low value of the accessible scattering vector $\left(0.05 \AA^{-1}\right)$ and second, the particular specimen was subjected to a single field value along the $\perp^{r}$ direction.

In this paper we report on the investigation of reinduction of the untrained state in a $\mathrm{Co} / \mathrm{CoO} / \mathrm{Au}$ multilayer using different values of the reorientation field $\left(H_{\mathrm{RE}}\right)$ and along three different orientations $\left(\Omega_{\mathrm{RE}}\right)$ with respect to the cooling field direction. Simulations have indicated earlier that the reinduction of the untrained state depends on the amplitude and direction of the reorientation field. We have done a detailed investigation of the phenomena by measuring the parallel component of magnetization using a superconducting quantum interference device (SQUID). Furthermore, using PNR we have investigated the parallel as well as the perpendicular components of magnetization for each representative case identified in the SQUID data.

\section{SAMPLES AND MEASUREMENTS}

We have investigated multilayers of the composition $\mathrm{SiO}_{2} /[\mathrm{Co}(13.0 \mathrm{~nm}) / \mathrm{CoO}(5.0 \mathrm{~nm}) / \mathrm{Au}(20.0 \mathrm{~nm})]_{16}$ prepared by dc magnetron sputtering [10]. During deposition, the Ar pressure in the magnetron sputtering chamber was $3 \times 10^{-3}$ mbar. The process was started at a base pressure of $1 \times 10^{-7}$ mbar. We employ an ultraviolet light assisted oxidation at an $\mathrm{O}_{2}$ pressure of $200 \mathrm{mbars}$ at $50^{\circ} \mathrm{C}$ for $1 \mathrm{~h}$ [4].

Microstructural characterization has been done using crosssectional transmission electron microscopy (XTEM). The samples were prepared by standard mechanical (diamond) polishing followed by $\mathrm{Ar}^{+}$ion milling at $4 \mathrm{kV}$ for about $1 \mathrm{~h}$. A conventional bright-field imaging mode was used.

Conventional in-plane magnetization loops are measured using a SQUID, MPMS from Quantum Design. All SQUID measurements were done after the sample was cooled down to $10 \mathrm{~K}$ from room temperature (RT) in the presence of a well defined cooling field of $H_{\mathrm{FC}}=+10.0 \mathrm{kOe}$ (along the $-y$ axis) provided by a cryomagnet and then it was subjected to two consecutive field cycling (first and second). After the second hysteresis loop we have completed a third hysteresis loop (which starts and ends in the $\Omega_{\mathrm{RE}}$ directions) before measuring the hysteresis loop along the fourth (and fifth) field cycle (which is along the same direction as the first and second field cycles). Thus, expect for the third field cycle, all measurements were along the $y$ axis.

The sample was raised to room temperature and cooled again each time we opt for a different amplitude of the reorientation field $H_{\mathrm{RE}}$ and also along a different orientation $\Omega_{\mathrm{RE}}$ in measuring along the third field cycle. The purpose of the third field cycle is merely to reorient the spin configuration after the first field cooling. The orientation angles were made possible by using a precision sample rotator attached to the SQUID.
The neutron scattering experiments were performed at the polarized neutron reflectometer with polarization analysis AMOR in a time of flight (TOF) mode at SINQ, Paul Scherrer Institute in Switzerland. The neutron measurements were done following the very same procedure as described for measuring the SQUID data which enabled us to measure along the decreasing branch (applied field opposite to the cooling field). The different orientation angles of the sample with respect to the initial cooling field direction were made possible by using a unique precision sample rotator within the cryomagnet at the sample position of AMOR.

Depth sensitive neutron scattering under grazing incidence with polarization analysis (PNR) [16-18] were used for our investigation on a microscopic scale. A detailed description of the PNR technique and measurements can be found in Refs. [16,19]. The reflectivity was recorded as a function of scattering vector,

$$
\begin{gathered}
\vec{Q}=\vec{k}_{i}-\vec{k}_{f}, \\
Q_{z}=Q_{\perp}=\frac{2 \pi}{\lambda}\left[\sin \left(\alpha_{\mathrm{i}}\right)+\sin \left(\alpha_{\mathrm{f}}\right)\right] \simeq \frac{2 \pi}{\lambda}\left[\alpha_{\mathrm{i}}+\alpha_{\mathrm{f}}\right], \\
Q_{x}=Q_{\|}=\frac{2 \pi}{\lambda}\left[\cos \left(\alpha_{f}\right)-\cos \left(\alpha_{i}\right)\right] .
\end{gathered}
$$

Here the incident wave vector defined by $\vec{k}_{i}$, makes an angle $\alpha_{\mathrm{i}}$ in the $x-z$ plane with respect to the $x$ axis while the scattered wave vector $\overrightarrow{k_{f}}$ makes angle $\alpha_{\mathrm{f}}$. In specular scattering geometry, the normal wave vector transfers $Q_{\perp}$ are probed while off-specular scattering contributions along the in-plane momentum transfer vector $Q_{\|}$arise, when the inplane translational symmetry is broken by interface waviness (roughness) or by magnetic domains on a length scale shorter than the neutron coherence length $\left(l_{\|}\right)$along $Q_{\|}$.

The nonspin flip (NSF) scattering amplitude provides information about $\rho_{\mathrm{n}} \pm \rho_{\mathrm{m}} \cos \phi_{\mathrm{A}}$, and the spin flip (SF) channels measure $\rho_{\mathrm{m}}^{2} \sin ^{2} \phi_{\mathrm{A}}$, if the domain size is larger than the projection of the neutron coherence length along the sample plane $\left(l_{\|}\right)$. Here, $\rho_{\mathrm{n}}$ is the nuclear and $\rho_{\mathrm{m}}$ is the magnetic scattering length density (SLD). We designate $\phi_{\mathrm{A}}$ as the angle between the direction of FM magnetization $\left(\mathbf{M}_{\mathbf{F M}}\right)$ and the neutron spin quantization axis. The neutron polarization vector is guided by the field applied to the sample $\left(H_{\mathrm{a}}\right)$ along the $y$ axis.

With spin analysis of the reflected neutron beam, it is possible to measure independently the NSF and SF reflectivities. PNR is sensitive to the directions of the parallel $\left(\mathbf{M}_{\mathbf{F M} \|}\right)$ and perpendicular $\left(\mathbf{M}_{\mathbf{F M} \perp}\right)$ components of the magnetic moment. In the experiment three different cross sections were distinguished, namely NSF channels : $\left(R_{++}\right.$and $\left.R_{--}\right)$and SF channel : $\left(R_{-+}\right)$. Here + and - signs are used to distinguish the intensity contributions $R$ representing the polarization component $\|$ and anti- $\|$ to the guiding field, respectively. $R_{++/--}$contains the sum/difference between $\rho_{\mathrm{n}}$ and $\rho_{\mathrm{m}}$, whereas the SF signal contains only the magnetic information via $\rho_{\mathrm{m}}$

The NSF reflectivities involve squares of the combinations of $\left(1-\cos \phi_{\mathrm{A}}\right)$ and $\left(1+\cos \phi_{\mathrm{A}}\right)$ terms while $\mathrm{SF}$ reflectivities involve $\left(\sin ^{2} \phi_{\mathrm{A}}\right)$ term. Thus, within the one dimensional 


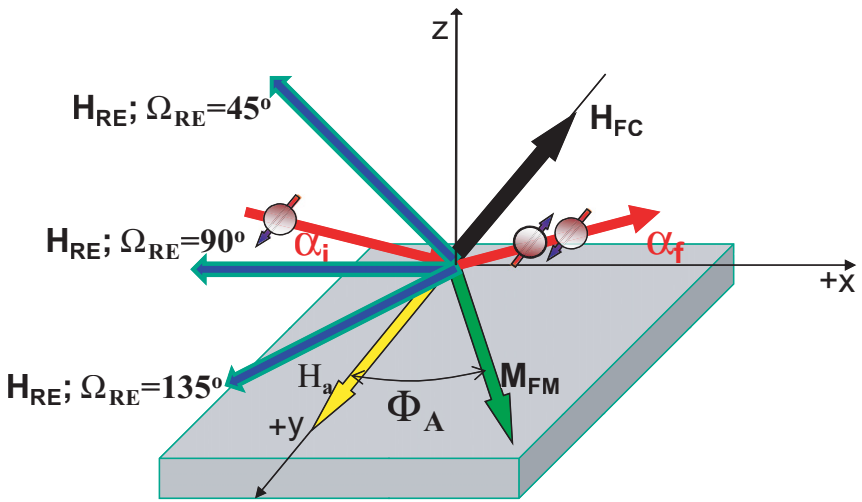

FIG. 1. (Color online) Illustration of the neutron scattering geometry. $H_{\mathrm{a}}$ along the $+y$ axis is shown to be antiparallel to $H_{\mathrm{FC}} \cdot M_{\mathrm{FM}}$ is the FM magnetization making an angle $\Phi_{A}$ with respect to the field axis. The reorientation field $H_{\mathrm{RE}}$ along the $-x$ axis (for $\Omega_{\mathrm{RE}}=90^{\circ}$ ) is also shown along with two other orientations $\left(\Omega_{\mathrm{RE}}=45^{\circ}\right.$ and $\left.135^{\circ}\right)$ in the sample plane.

analysis of the polarization vector (for noncollinear structures) it is not possible to discriminate the tilt angle $\phi_{\mathrm{A}}$ from $\left(\phi_{\mathrm{A}}+\pi\right)$ [16].

Figure 1 shows a sketch of the neutron scattering geometry. Here the applied field $H_{\mathrm{a}}$ along the $y$ axis is shown to be antiparallel to the cooling field $H_{\mathrm{FC}}$. $\mathbf{M}_{\mathbf{F M}}$ is making an angle $\Phi_{\mathrm{A}}$ with respect to the field axis. The reorientation fields $H_{\mathrm{RE}}$ are shown along different orientations $\Omega_{\mathrm{RE}}$ in the sample plane.

\section{RESULTS AND DISCUSSIONS}

\section{A. Microstructural measurements}

Figure 2 shows the XTEM micrographs of the sample. A multilayer structure with three different interfaces can be seen. The sample has a repeated sequence of around $21 \mathrm{~nm}$ of $\mathrm{Au}$, $11 \mathrm{~nm}$ of $\mathrm{Co}$ and $3.5 \mathrm{~nm}$ of $\mathrm{CoO}$ layers in the stack. These thicknesses are close to their nominal values.

\section{B. Magnetization measurements}

Figures 3, 4, and 5 show the SQUID hysteresis loops measured at $10 \mathrm{~K}$ for an in-plane cooling field. The parallel component of magnetizations $\left(\mathbf{M}_{\mathbf{F M} \|}\right)$ were measured for different strengths of the $H_{\mathrm{RE}}$ values and for three different orientation angles $\Omega_{\mathrm{RE}}$.

Clearly seen is the usual asymmetry in the magnetization reversal during the first field cycle (black curve) and the disappearance of the asymmetry during the second field cycle (red curve). We point out that the exchange bias field along the cooling field axis is estimated to be around -630 and -430 Oe for the first and second field cycles, respectively. This decrease in the bias field is due to commonly observed training. The first hysteresis loop shows a kink around -1500 Oe along the decreasing branch and at around +100 Oe along the increasing branch. These are typical indications of different oxidation levels in the CoO layers in the stack. Such a variation of oxidation affects the exchange coupling that results in a decrease in the switching fields along the respective branches [17].

After the first two field cycles the third hysteresis loop (magenta curve) shows the magnetization for different values of $H_{\mathrm{RE}}$ while completing a field cycle along $\Omega_{\mathrm{RE}}=45^{\circ}, 90^{\circ}$, and $135^{\circ}$ in Figs. 3, 4, and 5, respectively. At $\Omega_{\mathrm{RE}}=135^{\circ}$ the magnetization goes negative for $H_{\mathrm{RE}}=0.5 \mathrm{kOe}$ since it changes its direction. After each third field-cycling process, it was followed by two other consecutive hysteresis loop measurements along the fourth (blue curve) and fifth (green curve) field cycles, respectively.

Comparing the coercive fields for the second and fourth field cycles, one can find that there is indeed a reinduction of the untrained state. During the fifth field cycle, however, the revival is completely lost. The degree of reinduction of course varies with the strength of the $H_{\mathrm{RE}}$ values and with the angle $\Omega_{\mathrm{RE}}$.

Next we plot the derivative of the magnetization $\chi_{\text {mag }}=$ $d M / d H_{a}$ as a function of field corresponding to the different loops as shown in Fig. 6 for $H_{\mathrm{RE}}=0.5 \mathrm{kOe}$, as an example. The two peaks on either branch of the hysteresis loops indicate multiple switching fields. We neglect the smaller peak and
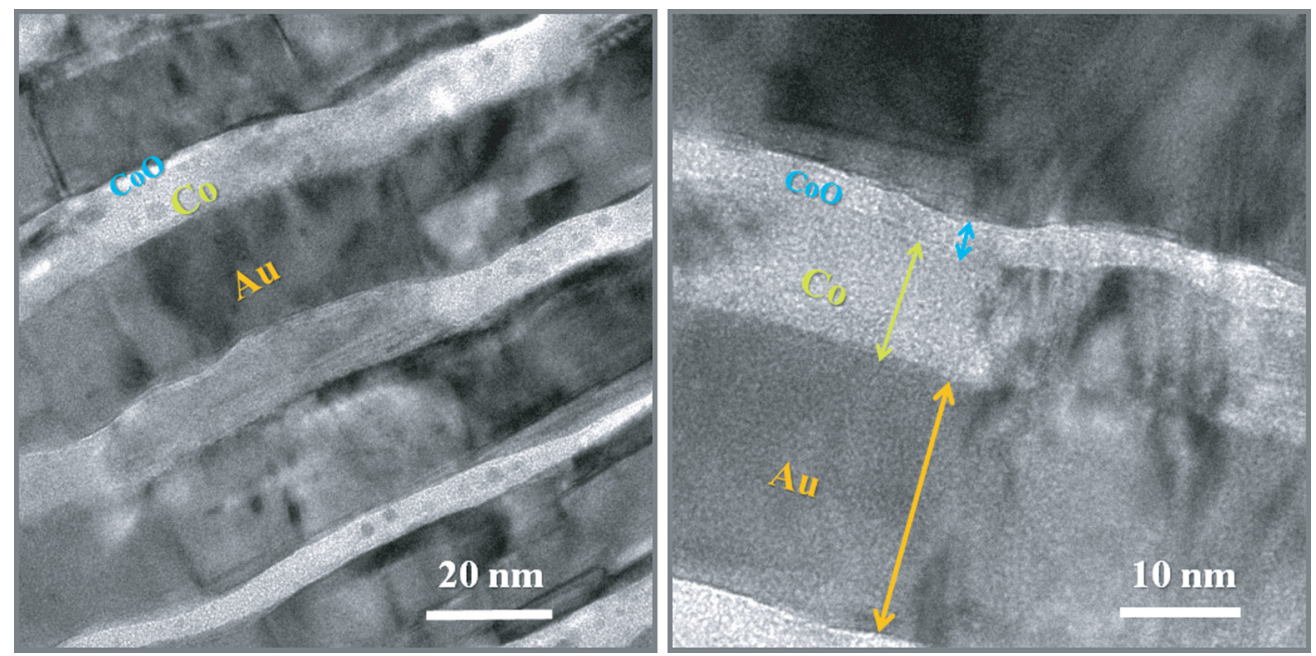

FIG. 2. (Color online) XTEM micrographs of the $[\mathrm{Co} / \mathrm{CoO} / \mathrm{Au}]_{16}$ multilayer. 

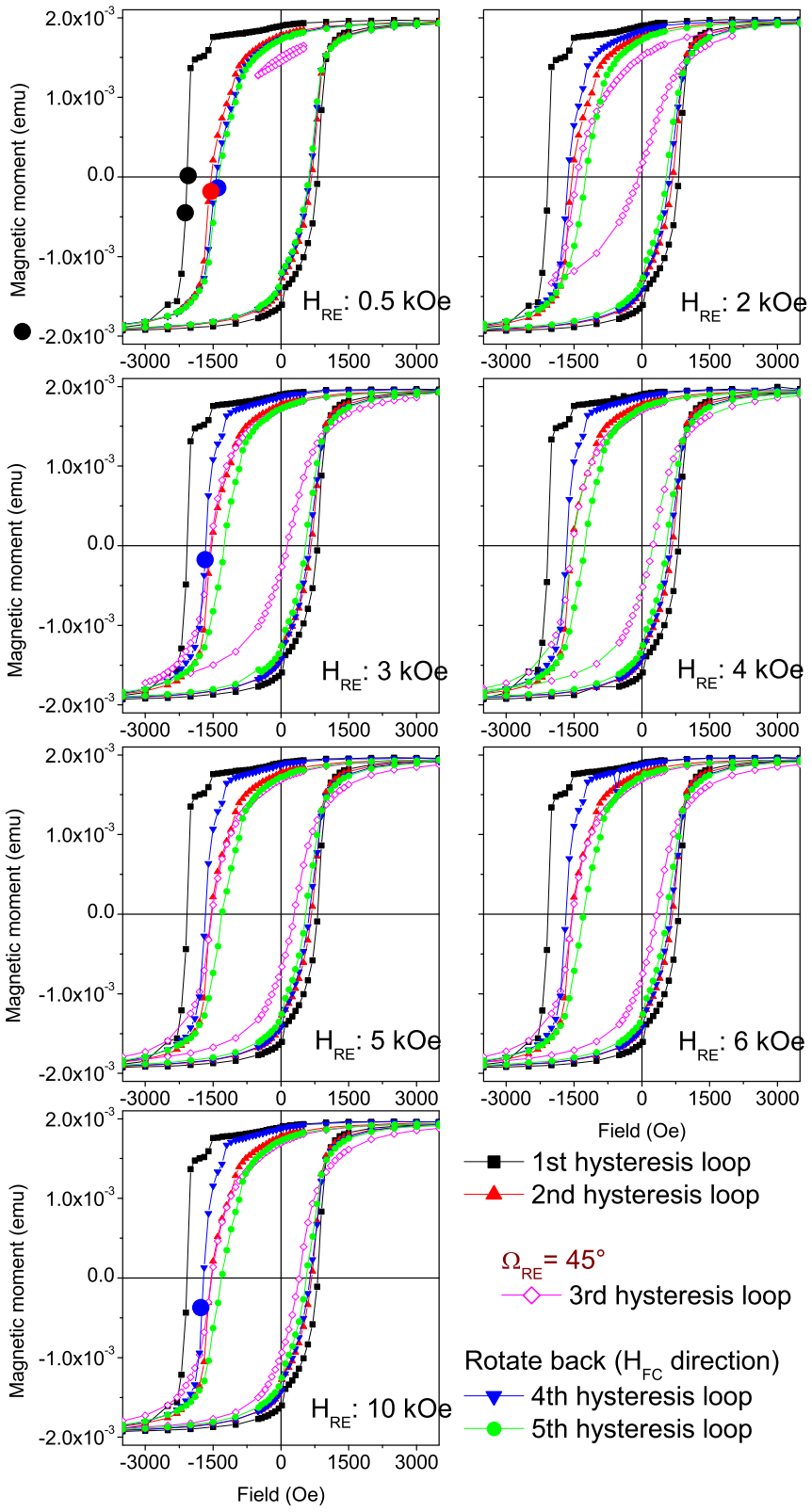

$\begin{array}{lll}-3000 & -1500 & 0 \\ & & \text { Field }(\mathrm{Oe})\end{array}$

- 1st hysteresis loop

- 2nd hysteresis loop

$\Omega_{\mathrm{RE}}=45^{\circ}$

$\checkmark$ 3rd hysteresis loop

Rotate back ( $\mathrm{H}_{\mathrm{FC}}$ direction)

$\nabla$ 4th hysteresis loop

- 5th hysteresis loop

FIG. 3. (Color online) SQUID magnetization hysteresis loops for different field cycles measured at $10 \mathrm{~K}$ and for various reorientation fields $H_{\mathrm{RE}}$ along $\Omega_{\mathrm{RE}}=45^{\circ}$ in the sample plane. The black, red, and blue circles mark the applied field values $H_{\mathrm{a}}$ for the neutron measurements along the respective branch of the loops.

concentrate on the main peaks A1 and A2 on the decreasing and the increasing branch, respectively, which represents most of the magnetization (from majority of the layers) within the layer stack.

In order to quantify the degree of reinduction of the untrained state, we have taken the following path. Firstly, we calculate the integrated areas under each peak from a Gaussian fit to each and every peak A1 and A2 for each field cycle shown in Fig. 6. Secondly, we estimate the degree of asymmetry by defining a parameter $\mathrm{X} 1$ for the first hysteresis loop as $\mathrm{X} 1=\mathrm{Hys}_{A 1} / \mathrm{Hys} 1_{A 2}$. A similar procedure was opted for the second, fourth, and fifth hys-
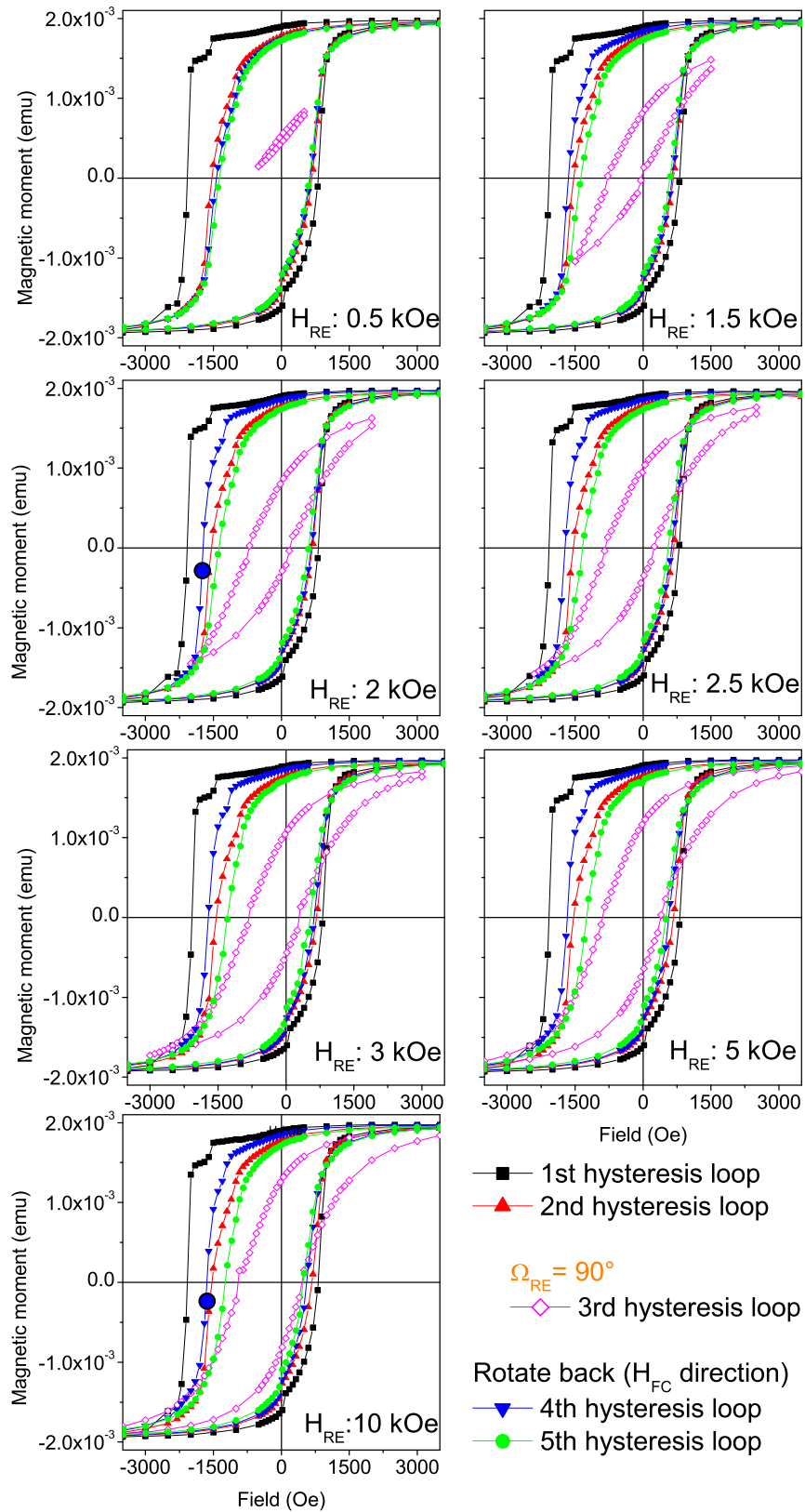

- 1 st hysteresis loop

- 2nd hysteresis loop

$\Omega_{\mathrm{RE}}=90^{\circ}$

$\diamond-3$ rd hysteresis loop

Rotate back $\left(\mathrm{H}_{\mathrm{FC}}\right.$ direction)

$\nabla-4$ th hysteresis loop

- 5 th hysteresis loop

FIG. 4. (Color online) SQUID magnetization hysteresis loops for different field cycles measured at $10 \mathrm{~K}$ and for various reorientation fields $H_{\mathrm{RE}}$ along $\Omega_{\mathrm{RE}}=90^{\circ}$ in the sample plane. The blue circles mark the applied field values $H_{\mathrm{a}}$ for the neutron measurements along the respective branch of the loops.

teresis loops as we estimate $\mathrm{X} 2=\mathrm{Hys} 2_{\mathrm{A} 1} / \mathrm{Hys} 2_{\mathrm{A} 2}, \mathrm{X} 4=$ Hys $_{\mathrm{A} 1} / \mathrm{Hys}_{\mathrm{A} 2}$, and X5 $=$ Hys $_{\mathrm{A} 1} / \mathrm{Hys}_{\mathrm{A} 2}$. Here Hys $1_{\mathrm{A} 1, \mathrm{~A} 2}$, $\mathrm{HSY} 2_{\mathrm{A} 1, \mathrm{~A} 2}, \mathrm{HSY} 4_{\mathrm{A} 1, \mathrm{~A} 2}$, and $\mathrm{HSY} 5_{\mathrm{A} 1, \mathrm{~A} 2}$ are the integrated areas under the peaks corresponding to the derivatives of the first, second, fourth, and fifth hysteresis loops, respectively. Finally, we plot in Fig. 7 the ratio of the integrated areas under each peak as $\mathrm{C} 12=\mathrm{X} 1 / \mathrm{X} 2, \mathrm{C} 14=\mathrm{X} 1 / \mathrm{X} 4$, and $\mathrm{C} 15=$ $\mathrm{X} 1 / \mathrm{X} 5$. Thus $\mathrm{C} 12$ (red up-triangles) lies close to unity and defines the initial degree of training with respect to the state where $H_{\mathrm{RE}}=0$ Oe and $\Omega_{\mathrm{RE}}=0^{\circ}$. This is compared with the case every time we heat the sample up to RT and cool down again to $10 \mathrm{~K}$ in $H_{\mathrm{FC}}=+10 \mathrm{kOe}$ as we set for a different value 

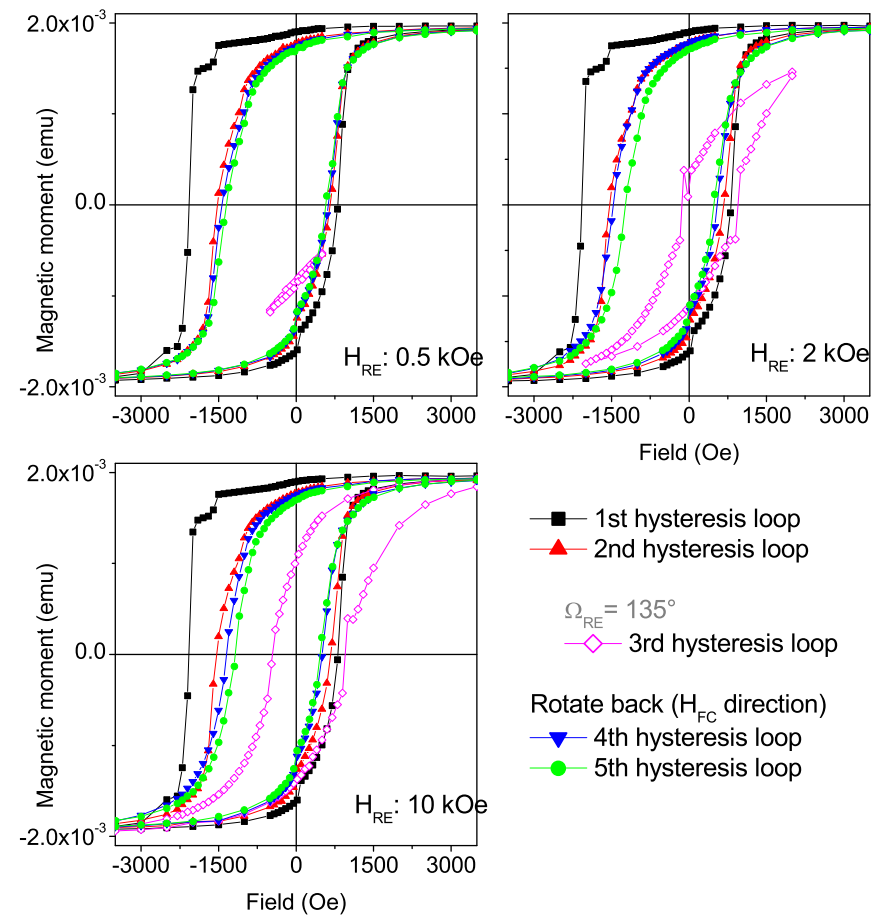

$$
\begin{aligned}
& -1 \text { st hysteresis loop } \\
& \Omega_{\mathrm{RE}}=135^{\circ} \\
& \curvearrowright-3 \text { rd hysteresis loop }
\end{aligned}
$$

Rotate back ( $\mathrm{H}_{\mathrm{FC}}$ direction)

$\checkmark$ 4th hysteresis loop

- 5th hysteresis loop

FIG. 5. (Color online) SQUID magnetization hysteresis loops for different field cycles measured at $10 \mathrm{~K}$ and for various reorientation fields $H_{\mathrm{RE}}$ along $\Omega_{\mathrm{RE}}=135^{\circ}$ in the sample plane.

of $H_{\mathrm{RE}}$ and $\Omega_{\mathrm{RE}}$. The slight deviation of $\mathrm{C} 12$ from unity with increasing $H_{\mathrm{RE}}$ redefines the initial trained state.

Let us now look into some specific cases. It may be noted that on the one hand while $\mathrm{C} 14$ (blue squares) is distinctly higher than $\mathrm{C} 12, \mathrm{C} 15$ (green circles) is always lower. This is

true for $\Omega_{\mathrm{RE}}=45^{\circ}$ and $\Omega_{\mathrm{RE}}=90^{\circ}$ and for all $H_{\mathrm{RE}}$ with an exception for $H_{\mathrm{RE}}=0.5 \mathrm{kOe}$, where $\mathrm{C} 14$ is also lower. On the other hand, for $\Omega_{\mathrm{RE}}=135^{\circ}, \mathrm{C} 14$ and $\mathrm{C} 15$ are always lower than $\mathrm{C} 12$. Following these plots one can infer on the fact that a reinduction of the training has plausibly taken place where $\mathrm{C} 14$ is higher than $\mathrm{C} 12$. The $\mathrm{C} 15$ plot indicates that the reinduction of training is eventually lost after such a revival.

To drive the point on the degree of reinduction/recovery further, we summarize our findings in Fig. 8 where we plot the ratio of the normalized integrated areas $\mathrm{C} 14$ with respect to $\mathrm{C} 12$ for the three $\Omega_{\mathrm{RE}}$ values. Here one can see that the reinduction is most efficient $(\sim 42 \%)$ for $\Omega_{\mathrm{RE}}=45^{\circ}$ and attains a fairly constant value after $H_{\mathrm{RE}}=3.0 \mathrm{kOe}$. For $\Omega_{\mathrm{RE}}=90^{\circ}$, one can see that a maximum of the reinduction is reached around $H_{\mathrm{RE}}=2.0 \mathrm{kOe}$. Note that this is very similar to the value reported by Brems et al. as well for the $\Omega_{\mathrm{RE}}=90^{\circ}$ case. This is in a way a vindication of our estimation procedure. Moreover, it agrees with their observation that the amplitude of the reorientation $(\perp)$ field needs to be sufficiently large $(\sim 1.9 \mathrm{kOe})$ in order to realize a maximum in reinduction [13]. Interestingly, unlike that in the $\Omega_{\mathrm{RE}}=45^{\circ}$ case, here we find a decrease (from $35 \%$ to $10 \%$ ) in the reinduction for any further increase in $H_{\mathrm{RE}}$. In the case when $\Omega_{\mathrm{RE}}=135^{\circ}$, however, there is no indication of reinduction.

\section{Neutron scattering measurements}

\section{First and second field cycles}

We show the specular NSF and SF scattering signals measured at $10 \mathrm{~K}$ in Figs. 9(a)-9(d) after the system has been cooled down in the presence of $H_{\mathrm{FC}}=+10.0 \mathrm{kOe}$. The data show at least four orders of magnitude drop in intensity at $Q_{\mathrm{z}}=0.11 \AA^{-1}$. Note that we have measured up to a high

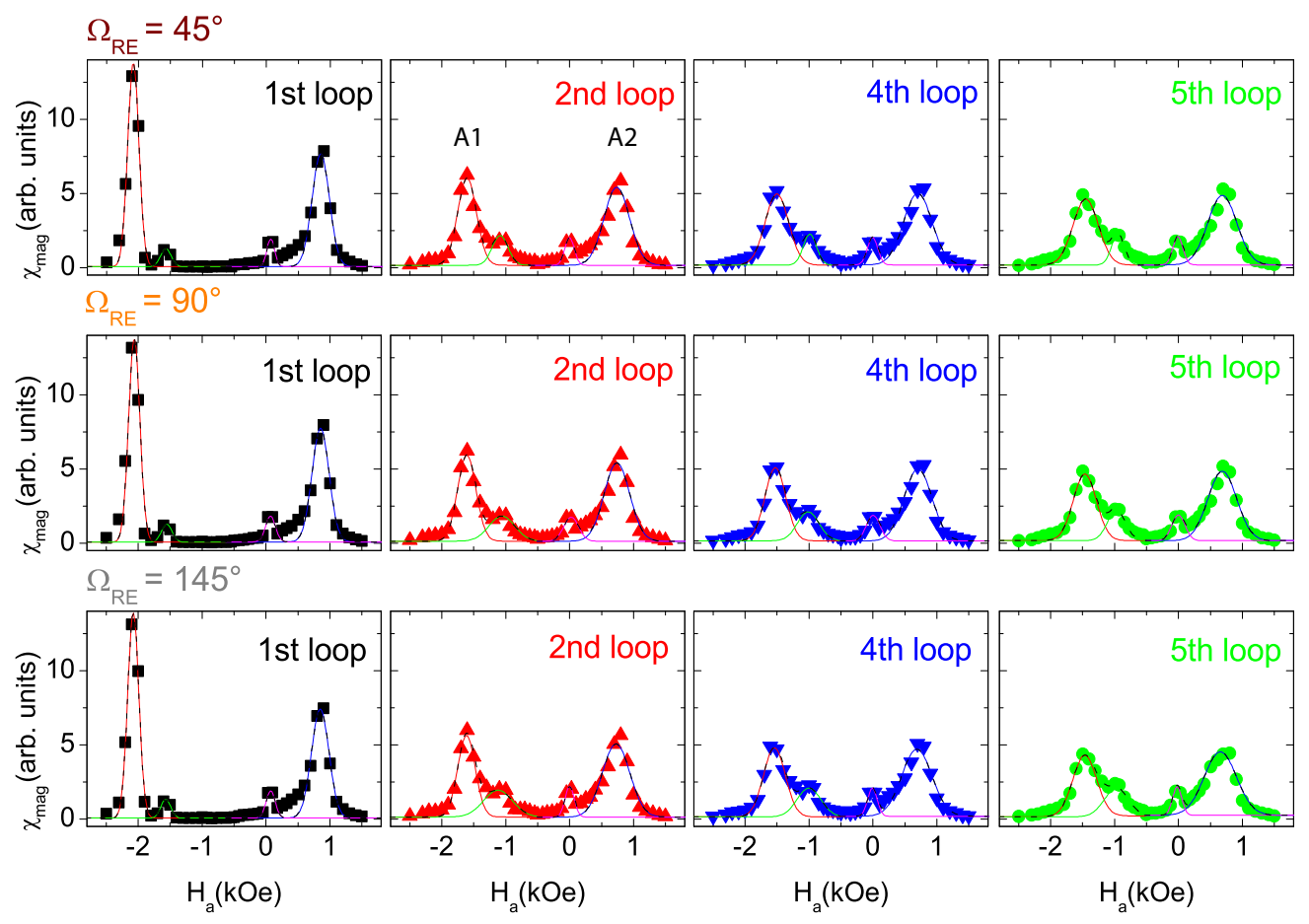

FIG. 6. (Color online) The field derivative of magnetization $\chi_{\mathrm{mag}}$ as a function of field measured at a reorientation field $H_{\mathrm{RE}}=0.5 \mathrm{kOe}$ and along different orientations $\Omega_{\mathrm{RE}}$ in the sample plane. The Gaussian fits to the peaks are shown in lines. 


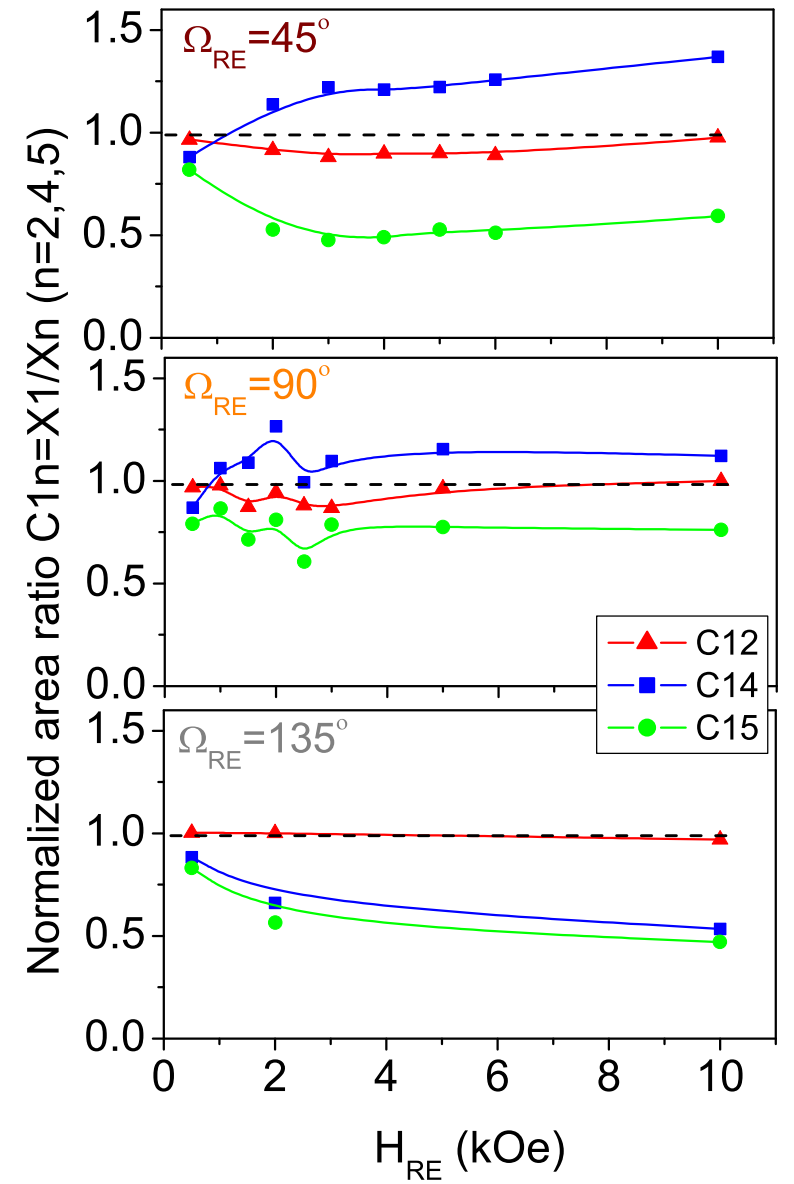

FIG. 7. (Color online) The normalized integrated area ratios $\mathrm{C} 1 n=\mathrm{X} 1 / \mathrm{X} n(n=2,4,5)$ with respect to the initial training for various reorientation field $H_{\mathrm{RE}}$ and along different orientations $\Omega_{\mathrm{RE}}$ in the sample plane. Here $n$ is the number of loop cycle. The symbol sizes are typical of their error bars.

value $\left(0.11 \AA^{-1}\right)$ of the scattering vector which is expected to reduce the possible ambiguities in the data analysis.

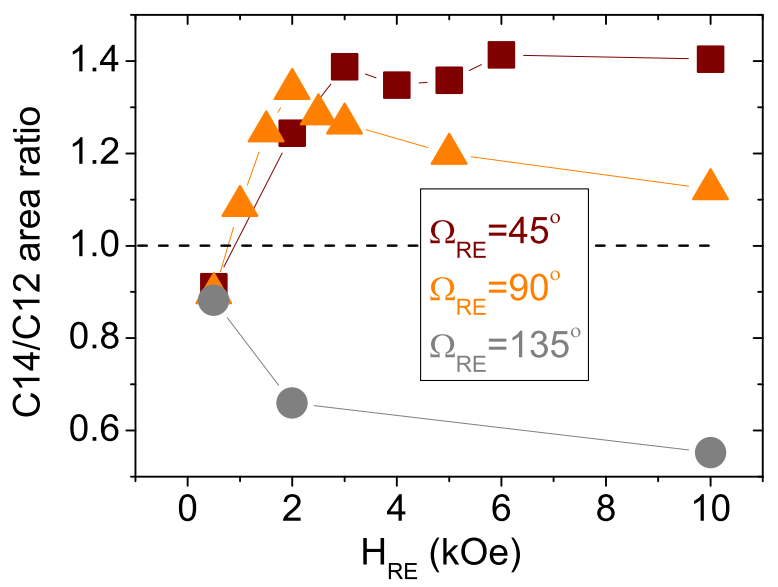

FIG. 8. (Color online) The ratio of normalized integrated area corresponding to the fourth field cycle with respect to the trained state during the fourth field cycle $(\mathrm{C} 14 / \mathrm{C} 12)$ for various reorientation field $H_{\mathrm{RE}}$ and along different directions $\Omega_{\mathrm{RE}}$ in the sample plane. The symbol sizes are typical of their error bars.
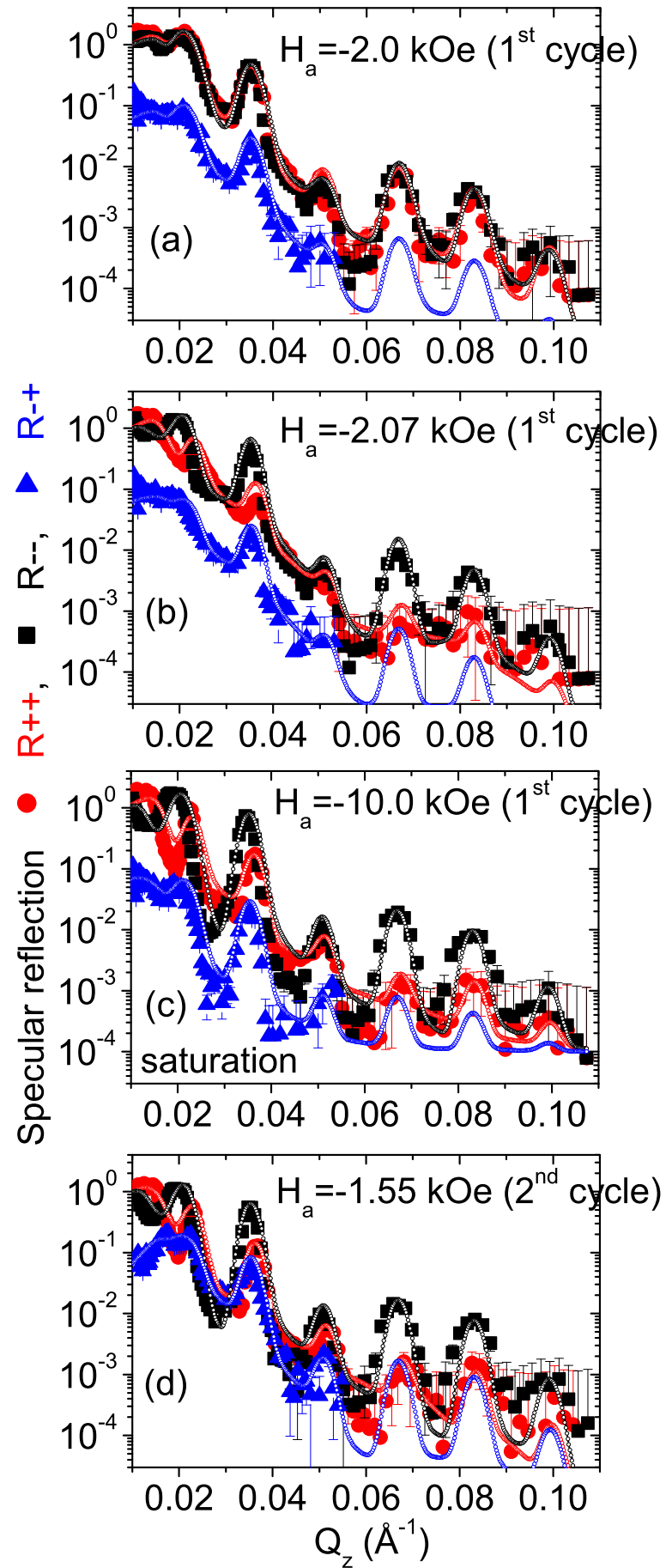

FIG. 9. (Color online) Specular reflectivity patterns (solid symbols) along with their best fits (open symbols) as a function of $Q_{\mathrm{z}}$ for the NSF [ $R_{--}$(black) and $R_{++}$(red)] and SF [ $R_{-+}$(blue)] channels measured at (a) close to the coercive field $H_{\mathrm{a}}=-2.0 \mathrm{kOe}$, (b) a slightly higher field $H_{\mathrm{a}}=-2.07 \mathrm{kOe}$, (c) a saturation field of $H_{\mathrm{a}}=-10.0 \mathrm{kOe}$ during the first field cycle, and (d) at $H_{\mathrm{a}}=-1.55$ kOe during the second field cycle.

Let us first concentrate on the data that was measured at a saturation field of $H_{\mathrm{a}}=-10.0 \mathrm{kOe}$ [Fig. 9(c)] during the first field cycle. The black circles in Fig. 3 show the fields of measurements. Since the data were measured at saturation we do not expect any SF signal to be present. The intensities 
TABLE I. Fit parameters extracted from the PNR results at saturation. Here $\rho_{\mathrm{n}}$ and $\rho_{\mathrm{m}}$ designate the nuclear and magnetic scattering length densities, respectively.

\begin{tabular}{lrrrrr}
\hline \hline Multilayer & \multicolumn{1}{c}{$\mathrm{Au}$} & $\mathrm{CoO}$ & $\mathrm{Co}$ & $\mathrm{Co}-\mathrm{Au}$ & Error \\
\hline Thickness $(\mathrm{nm})$ & 20.0 & 5.1 & 10.5 & 2.7 & \pm 0.2 \\
$\rho_{\mathrm{n}}\left(\times 10^{-6} \AA^{-2}\right)$ & 4.5 & 4.5 & 3.0 & 4.0 & \pm 0.2 \\
$\rho_{\mathrm{m}}\left(\times 10^{-6} \AA^{-2}\right)$ & 0.0 & 0.0 & 3.0 & 1.0 & \pm 0.1 \\
\hline \hline
\end{tabular}

in the SF channel are therefore from the small contribution of the NSF intensities which appears in the SF channels due to nonideal efficiencies of the polarizer and analyzer which amounts to a polarization efficiency factor $P \approx 94 \%$.

From the fits to the saturation data we get the informations on $\rho_{\mathrm{n}} \approx 3.0 \times 10^{-6} \AA^{-2}$ and $\rho_{\mathrm{m}} \approx 3.0 \times 10^{-6} \AA^{-2}$ for the Co layers in the stack. For the $\mathrm{Au}$ and $\mathrm{CoO}$ layers, $\rho_{\mathrm{n}} \approx$ $4.5 \times 10^{-6} \AA^{-2}$ was obtained. Additionally, we find a reduced magnetic layer $t_{\mathrm{Au}-\mathrm{Co}} \approx 2.7 \mathrm{~nm}$ with $\rho_{\mathrm{n}} \approx 4.0 \times 10^{-6} \AA^{-2}$ and $\rho_{\mathrm{m}} \approx 1.0 \times 10^{-6} \AA^{-2}$ at the Co-Au interface possibly due to canting as has been reported earlier in similar systems [17]. The thicknesses and the SLD values of the individual layers in the multilayer are found to be close to their nominal values and they are tabulated along with the nuclear and magnetic SLDs in Table I. Note that since $R_{--}>R_{++}$(as $H_{\mathrm{a}}$ is negative), we expect a complete alignment of the Co moments along the field direction at $-10.0 \mathrm{kOe}$, i.e., $\phi_{A}=0^{\circ}$.

Next we inspect the data measured at $H_{\mathrm{a}}=-2.0 \mathrm{kOe}$ [Fig. 9(a)] and $H_{\mathrm{a}}=-2.07 \mathrm{kOe}$ [Fig. 9(b)] during the first field cycle (marked by the black circles in Fig. 3). The fits to the data set reveal that the magnetization reversal is via domain wall and/or nucleation (DW) process when measured at a coercive field of $H_{\mathrm{a}}=-2.0 \mathrm{kOe}$. The identification of the reversal process via DW or rotational reversal from the SF signals was demonstrated more than a decade earlier [2]. Here in our model we have considered eight of the Co layers to have flipped $\left(\phi_{A}=0^{\circ}\right)$ along the field direction while the other eight layers have not $\left(\phi_{A}=180^{\circ}\right)$. The error bars in the turn angles are not more than $\pm 5^{\circ}$. The rotational sense of the magnetically weak intermediate layers $\left(t_{\mathrm{Co}-\mathrm{Au}}\right)$ has been always kept similar to that of the Co layers in the stack. However, due to the fact that the net magnetization in the system at this field is close to zero $\left(R_{++} \simeq R_{--}\right.$since $\left.\overline{\mathbf{M}_{\mathbf{F M}}}=\mathbf{0}\right)$ it is not possible to determine the preferential directional sense of the $\mathbf{M}_{\mathbf{F M}}$ with respect to the $H_{\mathrm{FC}}$ direction.

This scenario becomes somewhat different when the sample is measured at a slightly higher field. Then the directional sense of the FM layers can be clearly sensed. This is due to the imbalance in the torque on the magnetic layers that would be exerted on the layers at a field higher than the coercive field. Here at $H_{\mathrm{a}}=-2.07 \mathrm{kOe}$ [Fig. 9(b)], since $R_{--}>R_{++}$, one can infer from the fit to the data that more numbers of the FM layers (14 out of the 16 layers) have flipped $\left(\phi_{A}=0^{\circ}\right.$ ) towards the $H_{\mathrm{a}}$ direction while the bottommost layer $\left(\phi_{A}=180^{\circ}\right)$ has not. The top/bottom FM layer $\left(\phi_{A}=17^{\circ}\right)$ has a different turn angle which can be attributed to a loosely coupled state of the FM layer. The variation in coupling strength is due to the asymmetric sequence of the layers in the stack. A distinction

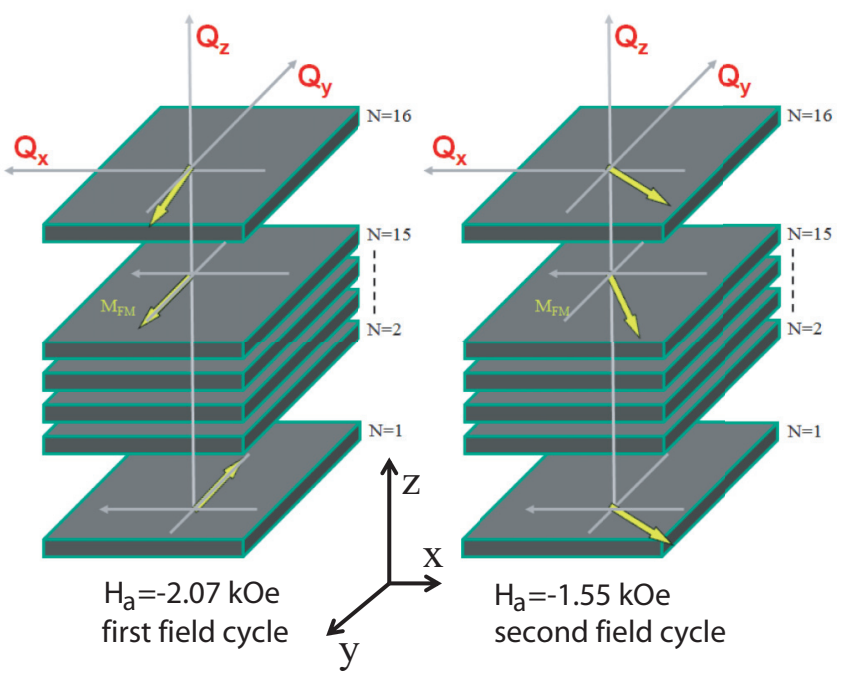

FIG. 10. (Color online) FM layer switching sequence during the first field cycle at $H_{\mathrm{a}}=-2.07 \mathrm{kOe}$ and during the second field cycle at $H_{\mathrm{a}}=-1.55 \mathrm{kOe}$. The arrows indicate the $\mathbf{M}_{\mathbf{F M}}$ orientations in the respective layers. The applied field $H_{\mathrm{a}}$ is along the $-y$ axis.

between the top or bottom layer upon their interchange in magnetization angle is not very clear thus the references to the top or bottom layers are merely for the sake of identification. It may be noted that the SF signals at the two measuring fields $\left(H_{\mathrm{a}}=-2.0\right.$ and $\left.-2.07 \mathrm{kOe}\right)$ did not show significant differences due to negligible variations in the perpendicular components of the respective layer magnetizations.

Next we inspect the data measured at $H_{\mathrm{a}}=-1.55 \mathrm{kOe}$ [Fig. 9(d)] during the second field cycle. The red circle in Fig. 3 shows the field of measurement. In this case we find from the fits to the data that almost all the FM layers (14) in the stack are undergoing a simultaneous rotational reversal process with $\phi_{A}=30^{\circ}$. Here again the topmost and the bottommost layer have slightly different turn angles $\left(\phi_{A}=50^{\circ}\right)$ from the rest.

In Fig. 10 we show the layer switching sequences for $H_{\mathrm{a}}=$ $-2.07 \mathrm{kOe}$ during the first field cycle and $H_{\mathrm{a}}=-1.55 \mathrm{kOe}$ during the second field cycle. The reversal is dominated by the DW process and by the rotational reversal process, respectively. The rotational senses of $\mathbf{M}_{\mathbf{F M}}$ for all the layers are seen to be preserved. Here the rotational senses of the layers are indicated towards the $H_{\mathrm{a}}$ direction as $R_{--}>R_{++}$. One may recall that apart from a decrease in the coercive field, rotational reversal process of the layers is also a strong signature of training in $\mathrm{Co} / \mathrm{CoO}$ systems where the untrained-state reversal mechanism during the first field cycle is usually via a DW reversal process.

In Fig. 11(a) we compare the measured SF scattering signals (perpendicular component of $M_{\mathrm{FM}}$ ) measured at $10 \mathrm{~K}$ and at a field of $H_{\mathrm{a}}=-2.07$ and $-10.0 \mathrm{kOe}$ (at saturation) during the first field cycle and $-1.55 \mathrm{kOe}$ during the second field cycle. The corrected SF signals in Fig. 11(b) is the subtracted-off SF signal (due to the inefficiencies of the optical elements) at saturation. This can be approximated as the net SF signal assuming no SF signal to be present at saturation (neutron polarization is collinear with the magnetization direction). 


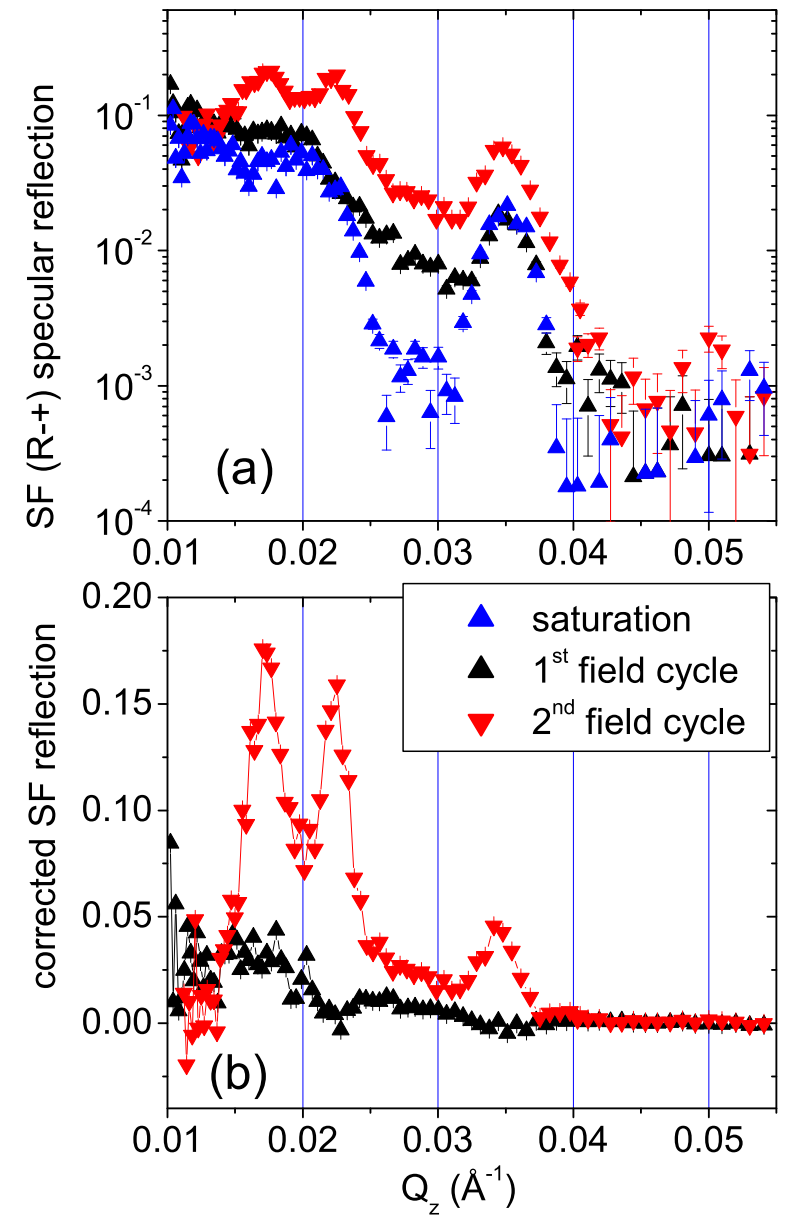

FIG. 11. (Color online) (a) SF specular reflectivity $\left[R_{-+}\right]$patterns as a function of $Q_{\mathrm{z}}$ measured during the first field cycle at $H_{\mathrm{a}}=-2.07$ and $-10.0 \mathrm{kOe}$ and during the second field cycle at $H_{\mathrm{a}}=-1.55 \mathrm{kOe}$. (b) Plot of the corresponding corrected SF signals as a function of $Q_{z}$.

The small increase in the corrected SF signal (black curve) during the first field cycle, which is unexpected during a DW reversal process, is due to some instability within one of the unflipped layers in the stack of 16 layers [18,20]. During the second field cycle (red curve) we find a significant increase in the corrected SF intensity. This increase is related to a rotational reversal process and thereby leads to training [2].

\section{Fourth field cycle with $\Omega_{\mathrm{RE}}=45^{\circ}$}

PNR measurements during the fourth field cycles were performed keeping $\Omega_{\mathrm{RE}}=45^{\circ}$. The blue circles in Fig. 3 show the fields of measurements. In Figs. 12(a)-12(c) we show the data measured at $10 \mathrm{~K}$ and at $H_{\mathrm{a}}=-1.44,-1.67$, and $-1.73 \mathrm{kOe}$, respectively during the fourth field cycles. The respective $H_{\mathrm{a}}$ values were chosen slightly higher than the coercive fields which are corresponding to the three different representative values of $H_{\mathrm{RE}}(=0.5,3.0$, and $10.0 \mathrm{kOe})$. Note that each time we chose to opt for a different $H_{\mathrm{RE}}$ value during the third field cycle, we have used a different $H_{\mathrm{a}}$ value during the fourth field cycle. This was done in accordance with the measured SQUID data.

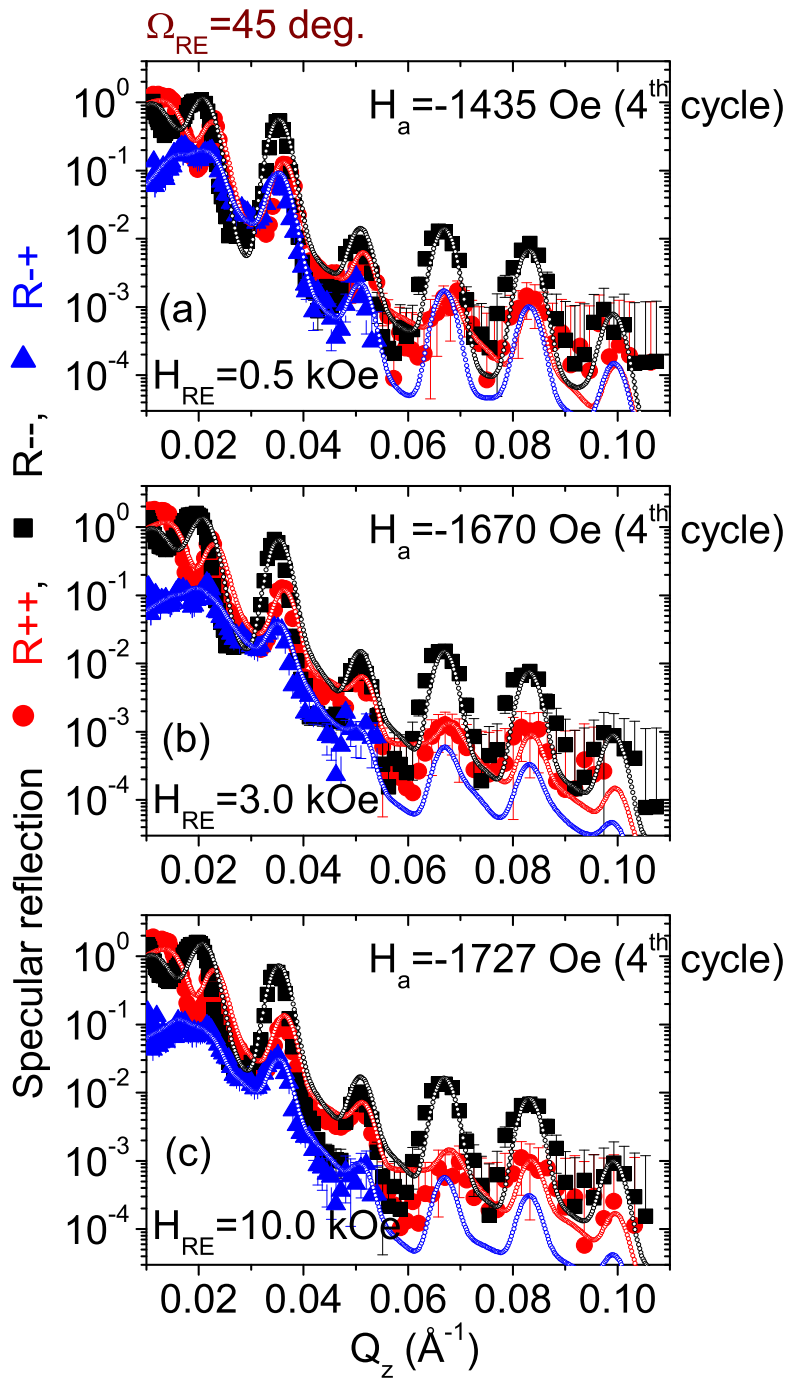

FIG. 12. (Color online) Specular reflectivity patterns (solid symbols) along with their best fits (open symbols) as a function of $Q_{\mathrm{z}}$ for the NSF $\left[R_{--}\right.$(black) and $R_{++}$(red)] and SF $\left[R_{-+}\right.$(blue)] channels measured at (a) $H_{\mathrm{a}}=-1.43 \mathrm{kOe}$, (b) $H_{\mathrm{a}}=-1.67 \mathrm{kOe}$, and (c) $H_{\mathrm{a}}=-1.73 \mathrm{kOe}$ during the fourth field cycle for different $H_{\mathrm{RE}}\left(=0.5,3.0\right.$, and $10.0 \mathrm{kOe}$, respectively) values when $\Omega_{\mathrm{RE}}=45^{\circ}$.

When $H_{\mathrm{RE}}=0.5 \mathrm{kOe}$, the fits to the data reveal magnetization reversal via rotation of the FM magnetization for majority of the layers with $\phi_{A}=35^{\circ}$. Here also we could find that almost all layers (14 out of the 16 layers) in the stack are rotating simultaneously with the exception of the top $\left(\phi_{A}=55^{\circ}\right)$ and the bottom $\left(\phi_{A}=65^{\circ}\right)$ layers which have different turn angles. The rotational senses of $\mathbf{M}_{\mathbf{F M}}$ for all the layers are seen to be preserved since $\phi_{A}$ lies in between $0^{\circ}$ and $180^{\circ}$.

In the case of $H_{\mathrm{RE}}=3.0 \mathrm{kOe}$, the fits to the data reveal magnetization reversal process of the multilayer is via a DW (flipping) or a rotational process within the individual layers. In this case, we find 12 out of the 16 layers with $\phi_{A}=0^{\circ}$ have flipped while the bottommost layer did not flip $\left(\phi_{A}=\right.$ $180^{\circ}$ ). Two layers from the top (with $\phi_{A}=25^{\circ}$ ) and a layer above the bottommost layer (with $\phi_{A}=40^{\circ}$ ) are found to be 


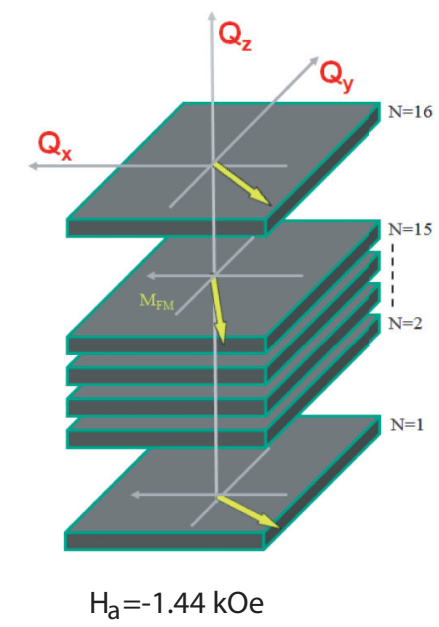

fourth field cycle $\mathrm{H}_{\mathrm{RE}}=0.5 \mathrm{kOe}$
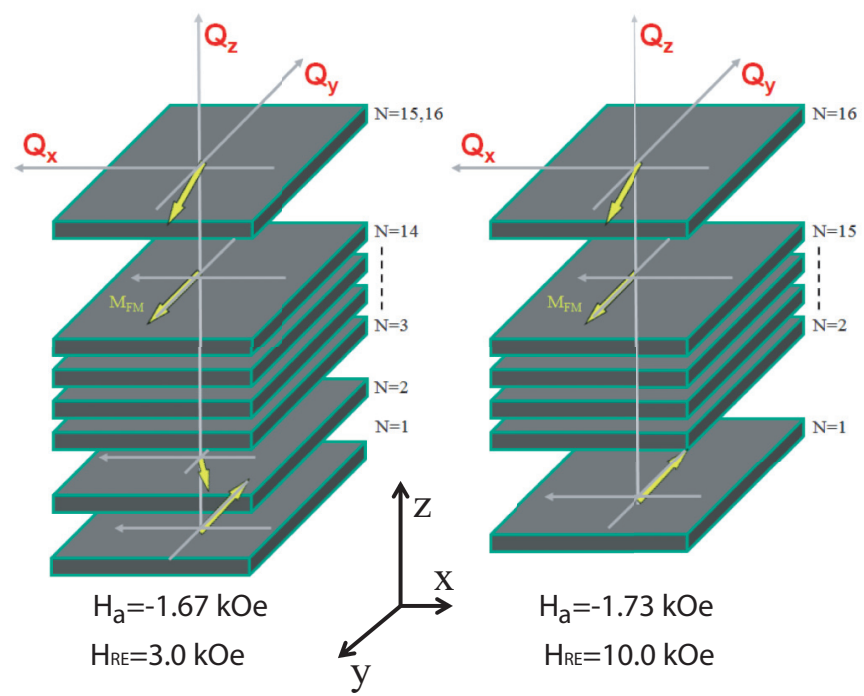

$\mathrm{H}_{\mathrm{a}}=-1.73 \mathrm{kOe}$

$\mathrm{H}_{\mathrm{RE}}=10.0 \mathrm{kOe}$

FIG. 13. (Color online) FM layer switching sequence during the different fourth field cycles at $H_{\mathrm{a}}=-1.44 \mathrm{kOe}\left(H_{\mathrm{RE}}=0.5 \mathrm{kOe}\right)$, $-1.67 \mathrm{kOe}\left(H_{\mathrm{RE}}=3.0 \mathrm{kOe}\right)$, and $-1.73 \mathrm{kOe}\left(H_{\mathrm{RE}}=10.0 \mathrm{kOe}\right)$ when $\Omega_{\mathrm{RE}}=45^{\circ}$. The arrows indicate the $\mathbf{M}_{\mathrm{FM}}$ orientations in the respective layers. The applied field $H_{\mathrm{a}}$ is along the $-y$ axis.

rotating. Thus, we have a combination of rotation and flipping, depending upon the degree of coupling of the layers.

For $H_{\mathrm{RE}}=10.0 \mathrm{kOe}$ we find a distinct layer-by-layer flipping scenario. The scenario is close to the case during the first field cycle. Here 13 out of the 16 layers have flipped $\left(\phi_{A}=\right.$ $0^{\circ}$ ), while the bottommost layer remain unflipped $\left(\phi_{A}=180^{\circ}\right)$. The top layer has a turn angle of around $\phi_{A}=25^{\circ}$.

In Fig. 13 we show the layer switching sequences for $H_{\mathrm{a}}=$ $-1.44,-1.67$, and $-1.73 \mathrm{kOe}$ during the respective fourth field cycles. Here we can see a gradual transition of the reversal process from being dominated by a rotational reversal process followed by a mixed process of rotational and DW reversal and finally taken over by a pure DW process.

In Fig. 14(a) we compare the measured SF scattering signals (perpendicular component of $M_{\mathrm{FM}}$ ) measured at $10 \mathrm{~K}$ and at fields of $H_{\mathrm{a}}=-1.44,-1.67$, and $-1.73 \mathrm{kOe}$ and during the respective fourth field cycles and compare them with $H_{\mathrm{a}}=$ $-10.0 \mathrm{kOe}$ (at saturation) during the first field cycle. The corrected SF signals in Fig. 14(b) is the subtracted-off SF signal at saturation.

We find a gradual decrease in the corrected SF signals (brown, dark yellow, and pink curves) with increasing $H_{\mathrm{RE}}$. The significant decrease in $\phi_{A}\left(=0^{\circ}\right.$ or $\left.180^{\circ}\right)$ for a majority of the layers in the case of $H_{\mathrm{RE}}=3.0$ and $10.0 \mathrm{kOe}$ as compared to that $\left(\phi_{A}=35^{\circ}\right)$ for $H_{\mathrm{RE}}=0.5 \mathrm{kOe}$, is mainly responsible for the decrease in the respective corrected SF signals.

One may note that in the case of $H_{\mathrm{RE}}=0.5 \mathrm{kOe}$, there is no flipping of the layers. Thus one cannot attribute this to reversal via a DW process. Since the rotational reversal process is a signature of training, one can readily infer that in this case there is no revival of the untrained state. This was also expected following the analysis of the SQUID data (Fig. 8).

When $H_{\mathrm{RE}}=3.0$, the decrease in the SF signal is due to the flipping of the layers which indicates a DW reversal mechanism for a majority of the layers. The remaining SF intensities, however, are due to the rotation of one or two top and bottom layers. Even though the reversal mechanism for
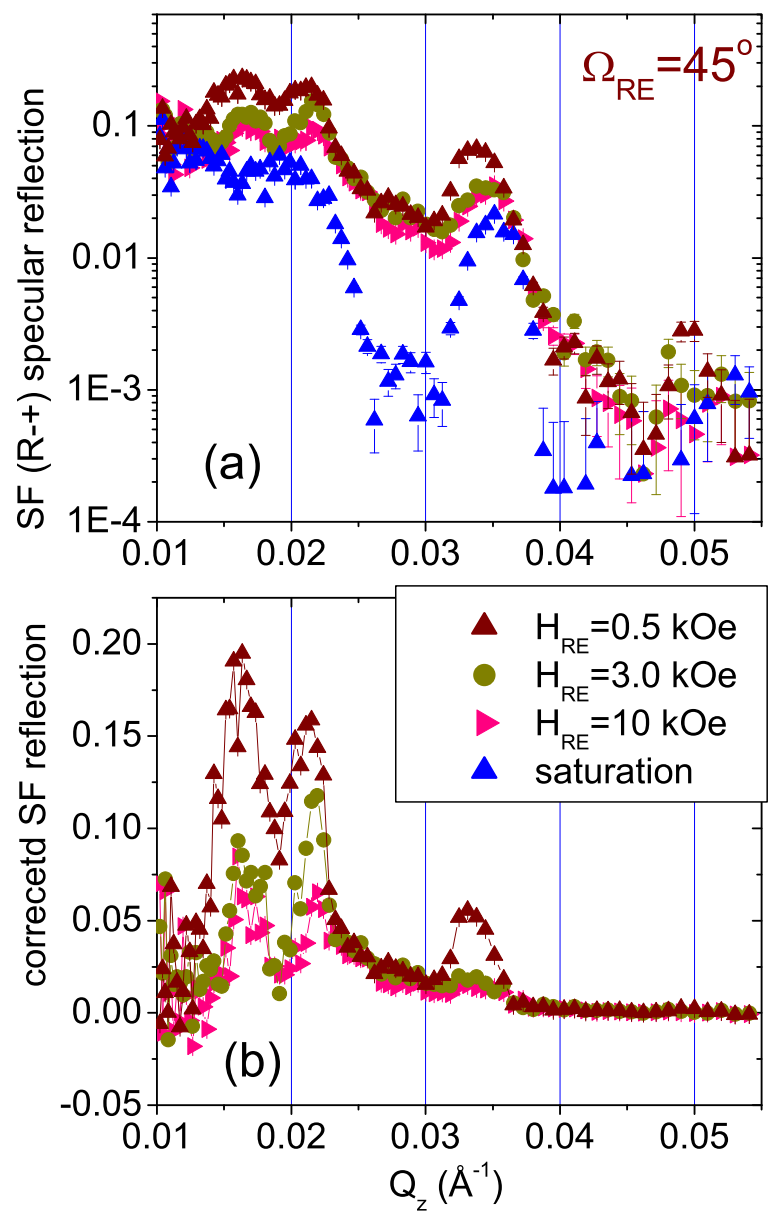

FIG. 14. (Color online) (a) SF specular reflectivity $\left[R_{-+}\right]$patterns as a function of $Q_{\mathrm{z}}$ measured during the fourth field cycle for $\Omega_{\mathrm{RE}}=45^{\circ}$ at $H_{\mathrm{a}}=-1.44,-1.67$, and $-1.73 \mathrm{kOe}$ for $H_{\mathrm{RE}}=0.5$, 3.0, and $10.0 \mathrm{kOe}$, respectively. (b) Plot of the corresponding corrected SF signals as a function of $Q_{\mathrm{z}}$. 
the majority of the layers is via a DW process, for the minority of the layers it is identified with rotation. Thus one can infer that the sample has signatures of mixed reversal processes. In other words, there is only partial revival of the untrained state.

Interestingly, in the case of $H_{\mathrm{RE}}=10.0 \mathrm{kOe}$, the reversal process is fully dominated via the DW process. Here one can infer that a significant revival of the untrained state has indeed taken place. One may also note that the orientations of the $\mathbf{M}_{\mathbf{F M}}$ do not depend upon the strength of $H_{\mathrm{a}}$, but upon the $H_{\mathrm{RE}}$ strength. Thus the degree revival of the untrained state has been shown to depend upon the $H_{\mathrm{RE}}$ strength.

\section{Fourth field cycle with $\Omega_{\mathrm{RE}}=90^{\circ}$}

PNR measurements during the fourth field cycles were performed keeping $\Omega_{\mathrm{RE}}=90^{\circ}$. The blue circles in Fig. 4 show the fields of measurements. In Figs. 15(a)-15(c) we show the data measured at $10 \mathrm{~K}$ and at $H_{\mathrm{a}}=-1.73$ and -1.65 $\mathrm{kOe}$, respectively, during the respective fourth field cycles corresponding to the two different representative values of $H_{\mathrm{RE}}(=2.0$ and $10.0 \mathrm{kOe}$ ). Here also each time we chose to opt for a different $H_{\mathrm{RE}}$ value during the third field cycle, we have used a different $H_{\mathrm{a}}$ value during the fourth field cycle. This was done again in accordance with the measured SQUID data.

In Fig. 16 we have shown the layer switching sequences for $H_{\mathrm{a}}=-1.73$ and $-1.65 \mathrm{kOe}$ during the respective fourth field cycles. Here one can see that for $H_{\mathrm{RE}}=2.0 \mathrm{kOe}$ the situation is typical of a magnetization reversal via the DW process.

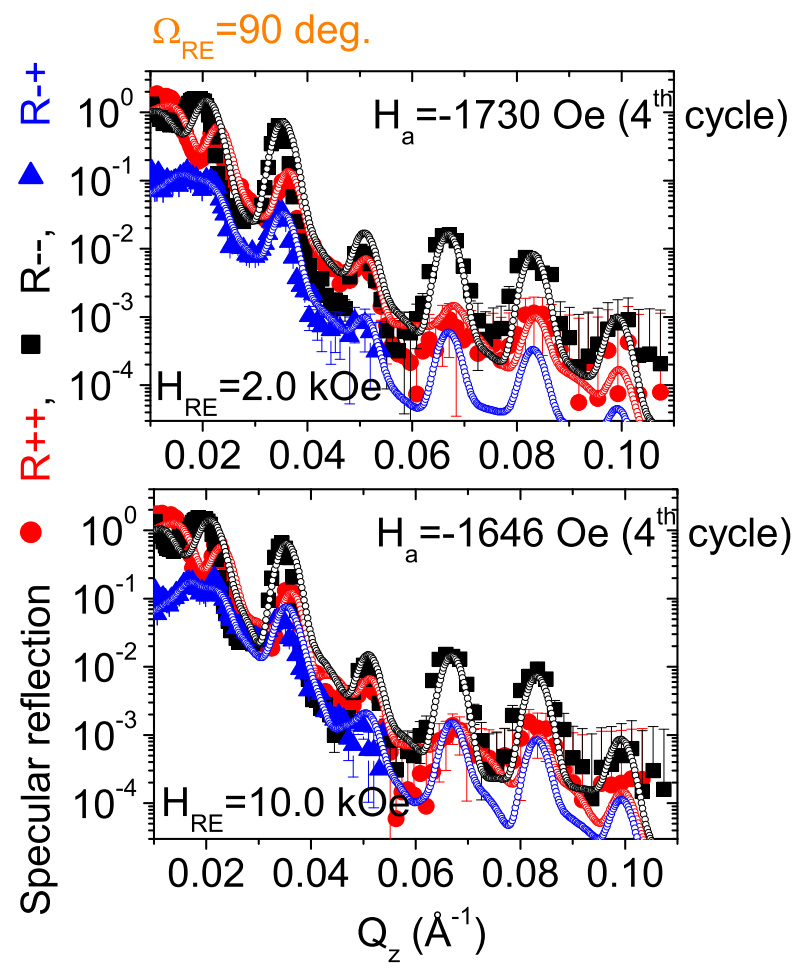

FIG. 15. (Color online) Specular reflectivity patterns (solid symbols) along with their best fits (open symbols) as a function of $Q_{\mathrm{z}}$ for the NSF $\left[R_{--}\right.$(black) and $R_{++}$(red)] and SF $\left[R_{-+}\right.$(blue)] channels measured at (a) $H_{\mathrm{a}}=-1.73 \mathrm{kOe}$ and (b) $H_{\mathrm{a}}=-1.65 \mathrm{kOe}$ during the fourth field cycle for different $H_{\mathrm{RE}}$ (=2.0 and $10.0 \mathrm{kOe}$, respectively) values when $\Omega_{\mathrm{RE}}=90^{\circ}$.

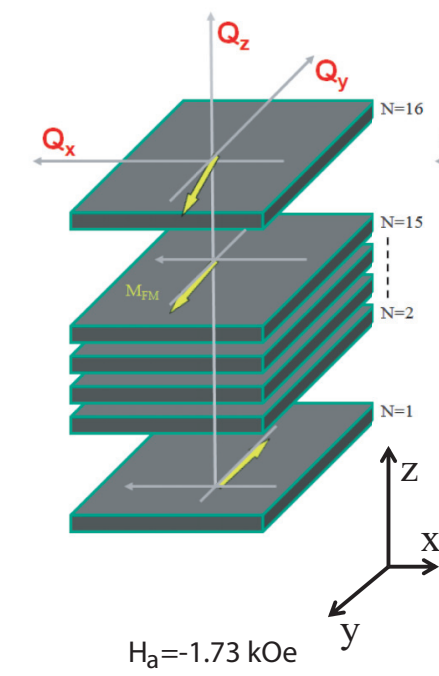

fourth field cycle $\mathrm{H}_{\mathrm{RE}}=2.0 \mathrm{kOe}$

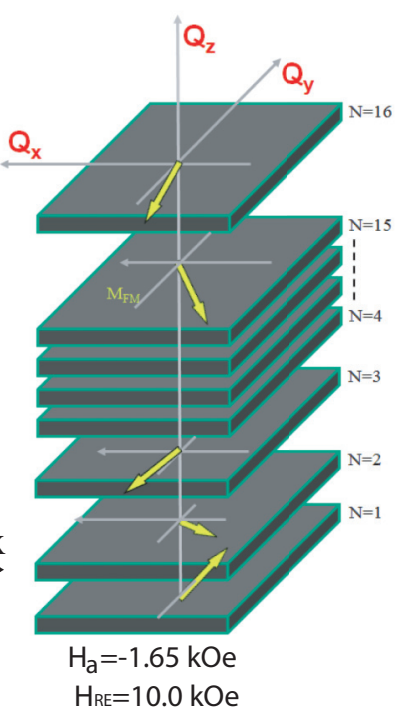

FIG. 16. (Color online) FM layer switching sequence during the different fourth field cycles at $H_{\mathrm{a}}=-1.73 \mathrm{kOe}\left(H_{\mathrm{RE}}=2.0 \mathrm{kOe}\right)$ and $-1.65 \mathrm{kOe}\left(H_{\mathrm{RE}}=10.0 \mathrm{kOe}\right)$ when $\Omega_{\mathrm{RE}}=90^{\circ}$. The arrows indicate the $\mathbf{M}_{\mathbf{F M}}$ orientations in the respective layers. The applied field $H_{\mathrm{a}}$ is along the $-y$ axis.

For $H_{\mathrm{RE}}=10.0 \mathrm{kOe}$, however, the situation is far more complicated. There is a mixture of DW and rotational reversal process. This is consistent with the earlier observations in similar systems by Brem et al. [11] as we can see that with further increase in the $H_{\mathrm{RE}}$ strength, the recovery of the untrained state is reduced.

In Fig. 17(a) we compare the measured SF scattering signals (perpendicular component of $M_{\mathrm{FM}}$ ) measured at $10 \mathrm{~K}$ and at fields of $H_{\mathrm{a}}=-1.73$ and $-1.65 \mathrm{kOe}$ during the respective fourth field cycles and at $H_{\mathrm{a}}=-10.0 \mathrm{kOe}$ (saturation) during the first field cycle. The corrected SF signals in Fig. 17(b) is the subtracted-off SF signal at saturation. The corrected SF signals can also be regarded as signatures of recovery or nonrecovery of the untrained state.

\section{Discussion on the rotational sense}

We can explain our experimental observations within the extended Fulcomer-Charap model [21], where a single ferromagnetic domain (FM magnetization) exchange couples with multiple AF grains with rotatable (responsible for training) and/or nonrotatable (responsible for bias field) moments but without any direct exchange coupling between the grains [22-24].

Brems et al. [13] have shown the consequences of changing the sense of direction during field cycling, for example, along $+x$ and $-x$ directions. The recovery of the untrained state depends upon the direction of the average uncompensated magnetization vector $\vec{m}_{\mathrm{AF}}$ of the antiferromagnetic grains. The directional sense of $\vec{m}_{\mathrm{AF}}$ was related to the directional sense of the FM magnetization. The whole sample magnetization was found rotating in the negative direction during the initial hysteresis loop which was accounted for in the initial negative direction of $\vec{m}_{\mathrm{AF}}$ as well [23,24]. 


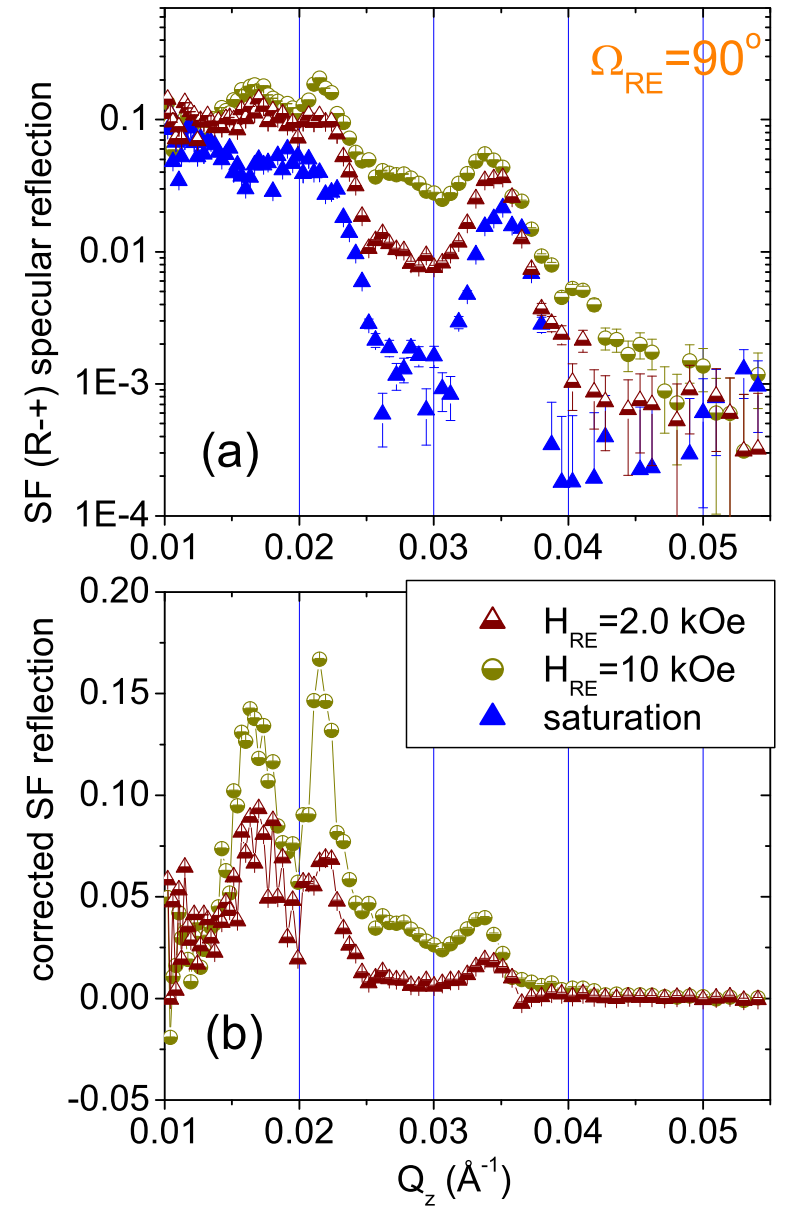

FIG. 17. (Color online) SF specular reflectivity $\left[\mathrm{R}_{-+}\right]$patterns as a function of $Q_{\mathrm{z}}$ measured during the fourth field cycle for $\Omega_{\mathrm{RE}}=90^{\circ}$ at $H_{\mathrm{a}}=-1.73 \mathrm{kOe}$, and $-1.65 \mathrm{kOe}$ for $H_{\mathrm{RE}}=2.0 \mathrm{kOe}$ and $10.0 \mathrm{kOe}$, respectively. (b) Plot of the corresponding corrected SF signals as a function of $Q_{\mathrm{z}}$.

The rotational sense of magnetization can be determined by following the trend of the angle $\phi_{A}$ with respect to the cooling field direction along the $-y$ axis for increasing measurement fields along the $+y$ axis. When the angle is positive (negative), the system undergoes a positive (negative) rotational sense. In a generic way, more is the angular direction of $\vec{m}_{\mathrm{AF}}$ oriented away from the cooling field direction, more is the torque acting on the FM moments. The torque in turn triggers magnetization reversal via rotation instead of the DW reversal process.

Let us now consider the case when $H_{\mathrm{RE}}$ is along the $+x$ axis $\left(\Omega_{\mathrm{RE}}=90^{\circ}\right)$ following the work of Brems et al. [13]. We show a sketch in Fig. 18, the direction of FM magnetization for $\Omega_{\mathrm{RE}}=90^{\circ}$ (started and ended in the $+x$ direction). Note that a maximum recovery of the untrained state was reported for $H_{\mathrm{RE}} \leqslant 1.9 \mathrm{kOe}$ (experimentally observed) only when the rotational sense (from $0^{\circ}$ to $-180^{\circ}$ ) remained unchanged with respect to the state realized after the initial field cooling. This was valid as long as $H_{\mathrm{RE}} \leqslant 1.9 \mathrm{kOe}$ [Fig. 18(a)]. For a $H_{\mathrm{RE}} \geqslant 1.9 \mathrm{kOe}$, the recovery was less pronounced since the rotational sense of the magnetization changed from negative to positive (i.e., from $180^{\circ}$ to $0^{\circ}$ ) with respect to the initial state after field cooling [Fig. 18(b)]. The recovery was also reported considerably less pronounced as the perpendicular

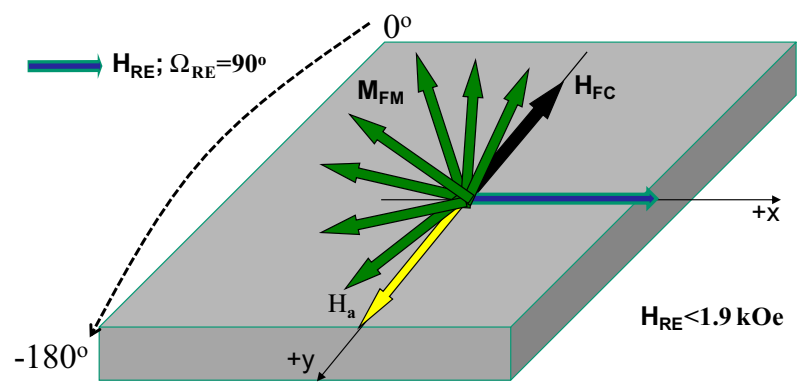

(a) negaitive rotation

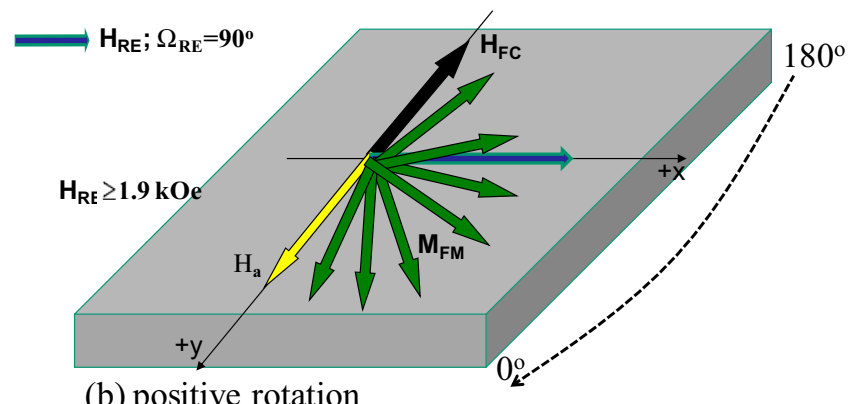

(b) positive rotation

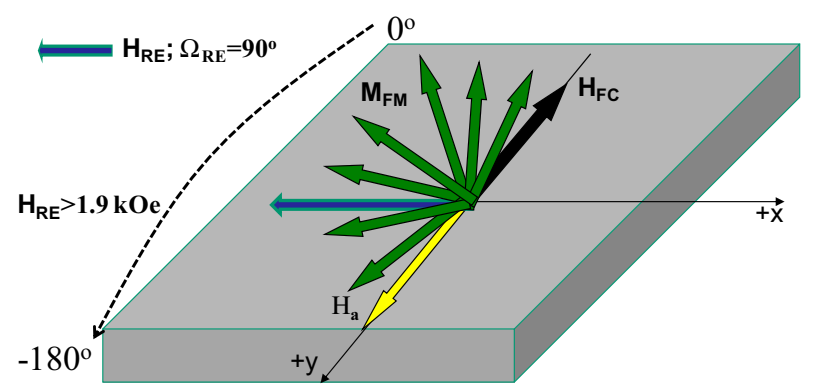

(c) negative rotation

FIG. 18. (Color online) Sketch showing the rotational sense of $\mathbf{M}_{\mathbf{F M}}$ under different conditions as $\Omega_{\mathrm{RE}}=90^{\circ}$ lies in the sample plane. The reorientation field $H_{\mathrm{RE}}$ can be either along the $+x$ axis (a) and (b) when the $\mathbf{M}_{\mathbf{F M}}$ rotational senses are sensitive to the strength of $H_{\mathrm{RE}}$ or along the $-x$ axis (c) when they are insensitive.

field started and ended in the $-x$ direction [Fig. 18(c)] and was insensitive to the value of $H_{\mathrm{RE}}$. Note that this was in spite of the fact that the rotational sense of the FM magnetization remained unchanged from the initial field cooled state.

The rotational sense of $\vec{m}_{\mathrm{AF}}$, which remains unknown from our experimental data, can be evaluated in principle by energy minimization of the total energy in the system. The total energy is given by

$$
\begin{aligned}
E= & \sum A^{i} k_{\mathrm{AF}} t_{\mathrm{AF}} \sin ^{2}\left(\phi^{i}-\alpha\right)-J_{\mathrm{int}} A^{i} \cos \left(\theta-\phi^{i}\right) \\
& -H_{\mathrm{a}} M_{\mathrm{FM}} t_{\mathrm{FM}} \cos (\chi-\theta) \sum A^{i} .
\end{aligned}
$$

Here the uniaxial anisotropy energy constant of $\mathrm{CoO}$ and $\mathrm{Co}$ are $k_{\mathrm{AF}}=2.5 \times 10^{8} \mathrm{erg} / \mathrm{cm}^{-3}$ and $k_{\mathrm{FM}}=1.5 \times 10^{6} \mathrm{erg} / \mathrm{cm}^{-3}$, respectively. $J_{\text {int }}$ is the interfacial exchange energy which involves the coupling constant between the FM and $\mathrm{AF}$ grains and $t_{\mathrm{FM}}\left(t_{\mathrm{AF}}\right)$ is the FM (AF) thickness. The angles are the respective angles made by $\vec{m}_{\mathrm{AF}}\left(\phi^{i}\right)$, AF easy axis $(\alpha)$, FM magnetization $(\theta)$, and $H_{\mathrm{a}}(\chi)$ with $H_{\mathrm{FC}}$ along the $-y$ axis. 


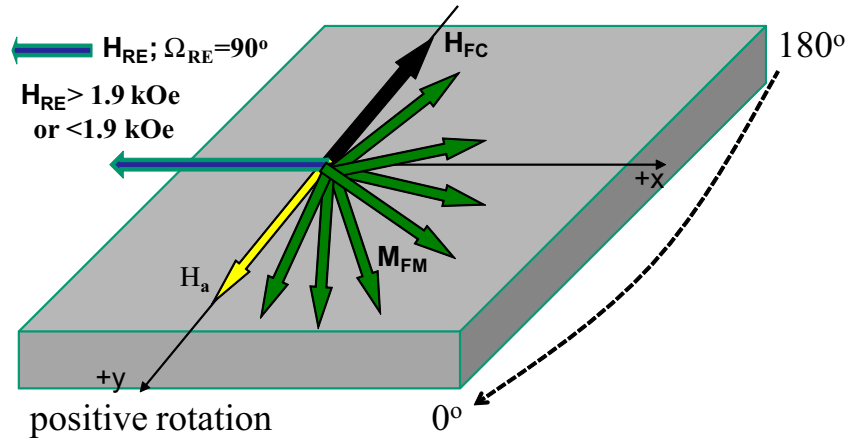

FIG. 19. (Color online) Sketch showing the rotational sense of $\mathbf{M}_{\mathbf{F M}}$ in the present geometry as $\Omega_{\mathrm{RE}}=90^{\circ}$ lies in the sample plane. The reorientation field $H_{\mathrm{RE}}$ is along the $-x$ axis when the $\mathbf{M}_{\mathbf{F M}}$ rotational sense is insensitive to the $H_{\mathrm{RE}}$ strength.

$A^{i}$ is the area of every noninteracting $\mathrm{AF}$ grain coupled to a single FM domain $\sum A^{i}$. We have considered 100 such AF grains coupled to one FM grain. We have neglected the term corresponding to $k_{\mathrm{FM}}$ as it is smaller by 2 orders of magnitude when compared with the value of $k_{\mathrm{AF}}$. From the value of $\rho_{\mathrm{m}}$ we estimate $M_{\mathrm{FM}}=1051 \mathrm{emu} / \mathrm{cm}^{3}$ or $1.25 \mu \mathrm{B} /$ atom, which gives $J_{\mathrm{int}}\left(=H_{\mathrm{eb}} M_{\mathrm{FM}} t_{\mathrm{FM}}\right)=0.86 \mathrm{erg} / \mathrm{cm}^{2}$.

For the first field cycling case, we can consider $\alpha=0^{\circ}$ assuming the $\mathrm{AF}$ easy axis to be coinciding with the $H_{\mathrm{FC}}$ axis after field cooling. Using the stability conditions $d E / d \phi^{i}=0$ and $d E / d \theta=0$ we are lead to a system of two equations:

$$
\begin{gathered}
k_{\mathrm{AF}} t_{\mathrm{AF}} \sin 2\left(\phi^{i}\right)+J_{\text {int }} \sin \left(\theta-\phi^{i}\right)=0, \\
H_{\mathrm{a}} M_{\mathrm{FM}} t_{\mathrm{FM}} \sin (\chi-\theta)+J_{\text {int }} \sin \left(\theta-\phi^{i}\right)=0 .
\end{gathered}
$$

In our case, since we always measure along the axis of $H_{\mathrm{FC}}$, we can consider $\chi=0^{\circ} / 180^{\circ}$ ( $\| /$ anti- $\|$ to the cooling axis). For finding the coercive fields we have used $\theta=\chi+\pi / 2$, considering the magnetization of the FM is zero across the easy axis which gives $\sin \phi^{i}=\frac{-J_{\text {int }}}{2 k_{\mathrm{AF}} t_{\mathrm{AF}}}$ or $\phi^{i}=-0.33^{\circ}$ from Eq. (5) during the first field cycle. Note that using a similar model, the average magnetization vector was calculated to be at $-0.6^{\circ}$ away from the cooling field direction by Brems et al. [11], signifying a negative sense of rotation.

In our case, using PNR alone, we cannot discriminate the rotational sense of the FM magnetization. In other words situations depicted in Fig. 18(c) cannot be discriminated from the situation depicted in Fig. 19. However, following the systematic variation of the magnetization directions (during the fourth field cycles) with applied fields $H_{\mathrm{a}}$, we could figure out that the rotational sense has not changed with the change in the $H_{\mathrm{RE}}$ values along a particular $\Omega_{\mathrm{RE}}$ direction. This unchanged FM rotational sense can be due to the fact that $H_{\mathrm{RE}}$ in our case always starts and ends in the $-x$ direction (at least for $\Omega_{\mathrm{RE}}=90^{\circ}$ ). This implies that in spite of an unchanged rotational sense of the FM magnetization, a well pronounced recovery of the untrained state is possible.

\section{SUMMARY AND CONCLUSION}

Antiferromagnetic-ferromagnetic exchange bias systems are known to suffer from training effect after the first field cycle. It has been shown that field cycling along a direction perpendicular to the field cooling direction can recover the untrained state, partially with different degrees of recovery. Here in this paper we have investigated a prototypical exchange - coupled multilayer in exploring the degree of recovery of the untrained state. We apply field cycling with various amplitudes $\left(H_{\mathrm{RE}}\right)$ and different orientation angles $\left(\Omega_{\mathrm{RE}}\right)$ with respect to the initial cooling direction. Systematic magnetization measurements by SQUID magnetometery along with depthsensitive vector magnetometry by PNR have been combined in our experiments.

Parallel component of magnetization measurements by SQUID indicate recovery of the untrained state for two orientation angles, namely $\Omega_{\mathrm{RE}}=45^{\circ}$ and $90^{\circ}$ along the $-x$ direction. PNR measurements, which simultaneously involve parallel as well as perpendicular components of magnetization, reveal that it is necessary to apply at least $H_{\mathrm{RE}}=3.0 \mathrm{kOe}$ to achieve a significant recovery. For a higher value of $H_{\mathrm{RE}}$ (up to $\approx 10.0 \mathrm{kOe}$ ), the recovery saturates while for a lower value, we could only achieve a partial recovery. For $\Omega_{\mathrm{RE}}=90^{\circ}$, we could reach a peak in the recovery even with $H_{\mathrm{RE}}=2.0 \mathrm{kOe}$. However, with higher fields, the recovery gradually decreases and only a partial recovery is possible. For any other higher angles of orientation (e.g., $\Omega_{\mathrm{RE}}=135^{\circ}$ ), no recovery of the untrained state is possible.

Our results are consistent with the earlier observations in similar systems [13] where a small degree of rotation with positive rotational sense (opposite to the sense after initial field cooling) of the AF magnetization vector-related to the FM magnetization direction - was attributed to partial recovery of the untrained state for $\Omega_{\mathrm{RE}}=90^{\circ}$. In our case we always observe an unchanged sense of the FM magnetization, irrespective of the strength and orientation of field cycling. The partial and/or significant recoveries can therefore be related to the directional sense of the AF magnetization vector. Contrary to the earlier observations, the degree of recovery is well pronounced in the present case, particularly for $\Omega_{\mathrm{RE}}=45^{\circ}$. Moreover, they can be regulated not only with the $H_{\mathrm{RE}}$ values but also with the $\Omega_{\mathrm{RE}}$ values below $90^{\circ}$. Thus we provide a deeper insight into understanding the fundamental mechanisms involved in the recovery of training. By lifting the directional restrictions of the field for the recovery of the untrained state, our work also opens up its technological prospects for such exchange biased systems, which remain coupled at room temperature.

\section{ACKNOWLEDGMENTS}

We would like to thank M. Braendlein for helping in the SQUID measurements and S. Mukherjee for his assistance during the PNR measurements. We would also like to thank $\mathrm{N}$. Paul and U. Bloeck for their assistance in the TEM measurements. This work is based on experiments performed at Amor at the Swiss spallation neutron source SINQ, Paul Scherrer Institute, Villigen, Switzerland. A.P. prepared the sample. A.S. did the SQUID measurements, and J.J. assisted A.P. in analyzing the data. J.J., J.S., and A.P. did the PNR measurements. All authors including P.E. and R.Y. read the manuscript. Discussions with Professor P. Böni are gratefully acknowledged. A.P. conceived and designed the work, analyzed the data, coordinated the work, and also wrote the manuscript. 
[1] W. H. Meiklejohn and C. P. Bean, Phys. Rev. 102, 1413 (1956).

[2] M. Gierlings, M. J. Prandolini, H. Fritzsche, M. Gruyters, and D. Riegel, Phys. Rev. B 65, 092407 (2002).

[3] A. Paul, E. Kentzinger, U. Rücker, and Th. Brückel, Phys. Rev. B 73, 092410 (2006).

[4] A. Paul, D. Bürgler, M. Luysberg, and P. Grünberg, Europhys. Lett. 68, 233 (2004).

[5] A. Paul, C. M. Schneider, and J. Stahn, Phys. Rev. B 76, 184424 (2007).

[6] A. Paul and S. Mattauch, Appl. Phys. Lett. 95, 092502 (2009).

[7] A. Paul, N. Paul, and S. Mattauch, New J. Phys. 13, 063008 (2011).

[8] M. Gierlings, M. J. Prandolini, M. Gruyters, T. Funk, D. Riegel, and W. D. Brewer, Eur. Phys. J. B 45, 137 (2005).

[9] M. Fecioru-Morariu, S. R. Ali, C. Papusoi, M. Sperlich, and G. Güntherodt, Phys. Rev. Lett. 99, 097206 (2007).

[10] A. Paul, E. Kentzinger, U. Rücker, and Th. Brückel, Phys. Rev. B 74, 054424 (2006).

[11] S. Brems, K. Temst, and C. Van Haesendonck, Phys. Rev. Lett. 99, 067201 (2007).

[12] S. Brems, D. Buntinx, K. Temst, C. Van Haesendonck, F. Radu, and H. Zabel, Phys. Rev. Lett. 95, 157202 (2005).

[13] S. Brems, H. Liu, K. Temst, and C. Van Haesendonck, Phys. Rev. B. 88, 214427 (2013).
[14] A. Paul, Appl. Phys. Lett. 97, 032505 (2010).

[15] A. Paul and S. Mattauch, Pramana J. Phys. 80, 701 (2013).

[16] H. Zabel, K. Th-Bröhl, and B. P. Toperverg, in Handbook of Magnetism and Advanced Magnetic Materials, edited by $\mathrm{H}$. Kronmüller and S. Parkin, Novel Techniques for Characterizing and Preparing Samples, Vol. 3 (John Wiley and Sons, New York, 2007).

[17] A. Paul, N. Paul, J. Jutimoosik, R. Yimnirun, S. Rujirawat, B. Höpfner, I. Lauermann, M. Lux-Steiner, S. Mattauch, and P. Böni, Phys. Rev. B 87, 014431 (2013).

[18] A. Paul, E. Kentzinger, U. Rücker, D. E. Bürgler, and P. Grünberg, Phys. Rev. B 70, 224410 (2004).

[19] C. F. Majkrzak and N. F. Berk, Phys. Rev. B 40, 371 (1989); A. Paul, Pramana J. Phys. 78, 1 (2012).

[20] A. Paul, T. Krist, A. Teichert, and R. Steitz, Physica B 406, 1598 (2011).

[21] E. Fulcomer and S. H. Charap, J. Appl. Phys. 43, 4190 (1972).

[22] T. Gredig, I. Krivorotov, and E. Dahlberg, J. Appl. Phys. 91, 7760 (2002).

[23] B. F. Miao, J. H. Ai, L. Sun, B. You, A. Hu, and H. F. Ding, Phys. Rev. B 82, 134442 (2010).

[24] J. Camarero, J. Sort, A. Hoffmann, J. M. García-Martín, B. Dieny, R. Miranda, and J. Nogués, Phys. Rev. Lett. 95, 057204 (2005). 